\title{
Thermal evolution and quiescent emission of transiently accreting neutron stars
}

\author{
A. Y. Potekhin ${ }^{1,2}$, A. I. Chugunov ${ }^{2}$, and G. Chabrier ${ }^{1,3}$ \\ 1 École Normale Supérieure de Lyon, CRAL (UMR CNRS 5574), 46 allée d’Italie, 69364 Lyon Cedex 07, France \\ 2 Ioffe Institute, Politekhnicheskaya 26, 194021 Saint Petersburg, Russia \\ e-mail: palex@astro.ioffe.ru \\ 3 School of Physics, University of Exeter, Exeter EX4 4QL, UK
}

Received 3 June 2019 / Accepted 17 July 2019

\begin{abstract}
Aims. We study the long-term thermal evolution of neutron stars in soft X-ray transients (SXTs), taking the deep crustal heating into account consistently with the changes of the composition of the crust. We collect observational estimates of average accretion rates and thermal luminosities of such neutron stars and compare the theory with observations.

Methods. We performed simulations of thermal evolution of accreting neutron stars, considering the gradual replacement of the original nonaccreted crust by the reprocessed accreted matter, the neutrino and photon energy losses, and the deep crustal heating due to nuclear reactions in the accreted crust. We also tested and compared results for different modern theoretical models. We updated a compilation of the observational estimates of the thermal luminosities in quiescence and average accretion rates in the SXTs and compared the observational estimates with the theoretical results.

Results. The long-term thermal evolution of transiently accreting neutron stars is nonmonotonic. The quasi-equilibrium temperature in quiescence reaches a minimum and then increases toward the final steady state. The quasi-equilibrium thermal luminosity of a neutron star in an SXT can be substantially lower at the minimum than in the final state. This enlarges the range of possibilities for theoretical interpretation of observations of such neutron stars. The updates of the theory and observations leave the previous conclusions unchanged, namely that the direct Urca process operates in relatively cold neutron stars and that an accreted heat-blanketing envelope is likely present in relatively hot neutron stars in the SXTs in quiescence. The results of the comparison of theory with observations favor suppression of the triplet pairing type of nucleon superfluidity in the neutron-star matter.
\end{abstract}

Key words. stars: neutron $-\mathrm{X}$-rays: binaries

\section{Introduction}

Neutron stars are the most compact stars ever observed: with typical masses $M \sim 1-2 M_{\odot}$, they have radii $R \approx 10-14 \mathrm{~km}$. The mass density $\rho$ in their core is $\sim 10^{15} \mathrm{~g} \mathrm{~cm}^{-3}$, several times the normal nuclear density (the typical density of a heavy atomic nucleus). Such dense matter cannot be obtained under laboratory conditions, and its properties and even composition remain to be clarified. Since these properties determine, in particular, the heat-loss rate of a neutron star, it is possible to study the extremely dense matter by comparing observed neutron-star surface luminosities with theoretical predictions (see, e.g., Potekhin et al. 2015 for review and references).

Many neutron stars reside in binary systems with a lowermass companion star (low-mass X-ray binaries, LMXBs) and accrete material onto their surfaces from the companion. In some cases, the accretion process is episodic. Such systems, called soft X-ray transients (SXTs), alternate between phases of accretion (outbursts), lasting usually days to months (sometimes years), and typically longer periods of quiescence. This transient activity is regulated most probably by the regime of accretion from the disks around the neutron stars (e.g., Lasota 2001). During an outburst, the X-ray emission of an LMXB is dominated by the accretion disk or a boundary layer (e.g., Inogamov \& Sunyaev 2010, and references therein). The released gravitational energy is so high that a transient looks like a bright X-ray source with luminosity $\sim\left(10^{36}-10^{38}\right) \mathrm{erg} \mathrm{s}^{-1}$. During quiescence, the accretion is switched off or strongly suppressed, and the luminosity decreases by several orders of magnitude (see, e.g., Wijnands et al. 2017, for review).

In spite of the increase of surface temperature to $\sim 10^{7} \mathrm{~K}$ during outburst, inflow of the heat generated by gravitational energy release is halted due to overheating of deeper layers by nuclear reactions associated with compression of the material (e.g., Fujimoto et al. 1984; Miralda-Escudé et al. 1990).

The neutron star core is predominantly heated by nuclear reactions occurring in the crust. When the accreted matter falls onto the neutron star, it pushes the underlying matter down to deeper layers and thus higher densities, where electron capture, neutron emission, and pycnonuclear reactions result in the deep crustal heating, with the release of $\sim 1-2 \mathrm{MeV}$ per accreted nucleon (Sato 1979; Haensel \& Zdunik 1990, 2003, 2008; Lau et al. 2018; Fantina et al. 2018). Eventually, the original ground-state "catalyzed" crust is replaced by a crust composed of accreted matter, while the original crust fuses with the core. Once an SXT turns to quiescence, thermal X-ray emission comes from the surface of the neutron star, meaning that the thermal relaxation of the crust can be observed directly (Brown et al. 1998; see, e.g., Wijnands et al. 2017 for a review).

We study the long-term thermal evolution of the SXTs, which determines the equilibrium level of their quiescent emission. This equilibrium can be reached after the post-outburst 
thermal relaxation of the crust if the relaxation lasts a sufficiently long time. For each neutron star, this level is a function of the temperature in the stellar core, which is controlled by the energy losses due to neutrino emission from the core and the crust and the photon emission from the surface balanced by the energy income due to the deep crustal heating, which is directly proportional to the accretion rate. Since the time needed for an appreciable heating or cooling of the core is much longer than the accretion variability (e.g., Colpi et al. 2001; Brown et al. 2018), the equilibrium level is a function of the average mass accretion rate $\langle\dot{M}\rangle$. Here and hereafter, the angle brackets $\langle\ldots\rangle$ denote averaging over a time-span covering many outburst and quiescence cycles. The dependence of the equilibrium luminosity on $\langle\dot{M}\rangle$ is called the heating curve (Yakovlev et al. 2003). Different neutron star models result in different heating curves, thus providing a means to check the models by comparison with observations.

The heating curves were previously calculated assuming that the initial ground-state crust is completely replaced by the reprocessed accreted matter (Yakovlev et al. 2003, 2004; Beznogov \& Yakovlev 2015a,b; Fortin et al. 2018; Matsuo et al. 2018). Meanwhile, it was noted that the accreted matter alone might be insufficient to fill the entire crust (e.g., Wijnands et al. 2013; Fantina et al. 2018). Here we perform self-consistent simulations of the long-term thermal evolution, considering the gradual replacement of the ground-state crust by an accreted one and the corresponding evolution of the heat release. We use a general-relativistic, implicit, adaptive-mesh finite-difference numerical code, which includes the most recent microphysics input (Potekhin \& Chabrier 2018; hereafter Paper I). We supplement the heating curves calculated for the fully accreted crust with the analogous curves that show the position of the minimum of the equilibrium luminosity of the SXTs on the long-term evolution curves, computed for the constant average accretion rate. The latter curves, together with the former ones, enlarge the range of equilibrium luminosities that correspond to a given average accretion rate and thus increase flexibility of the theory for explaining the observed equilibrium thermal luminosities of the SXTs in quiescence.

For the purpose of comparison of theory to observations, we revisit the average accretion rates and steady-state thermal luminosities of the neutron stars in SXTs in quiescence, evaluated from observations. We present a list of these properties for 35 SXTs, which includes relatively new observed SXTs as well as updates of the observational data traditionally used for comparison with the theoretical heating curves (e.g., Wijnands et al. 2017).

Finally, we explore the effect of suppression of the nucleon superfluidity by polarization (many-particle correlations), which is expected to be strong in the case of the triplet pairing gap (Ding et al. 2016; Sedrakian \& Clark 2018, and references therein). We show that it brings the theoretical heating curves in full accord with observations for all transiently accreting neutron stars, including those whose observed properties were only marginally compatible with the theory that ignored this effect.

\section{Physics input}

To model neutron-star cooling processes, we use the same physics input as in Paper I. This is briefly summarized below. In this section we also describe the additional ingredients of the physics input that are brought about by accretion and deep crustal heating.

\subsection{Equations of thermal evolution}

In the spherical symmetry, the thermal and mechanical structure of a star are governed by six first-order differential equations for radius $r$, gravitational potential $\Phi$, gravitational mass $M_{\mathrm{r}}$ inside a sphere of radius $r$, luminosity $L_{\mathrm{r}}$ passing through this sphere, pressure $P$, and temperature $T$ as functions of the baryon number $a$ interior to a given shell (Richardson et al. 1979; cf. Thorne 1977). Four equations determine the mechanical structure of the star,

$$
\begin{aligned}
& \frac{\mathrm{d} r}{\mathrm{~d} a}=\frac{1}{4 \pi r^{2} \bar{n}}\left(1-\frac{2 G M_{\mathrm{r}}}{r c^{2}}\right)^{1 / 2}, \\
& \frac{\mathrm{d} M_{\mathrm{r}}}{\mathrm{d} a}=\frac{\rho}{\bar{n}}\left(1-\frac{2 G M_{\mathrm{r}}}{r c^{2}}\right)^{1 / 2}, \\
& \frac{\mathrm{d} \Phi}{\mathrm{d} a}=G \frac{M_{\mathrm{r}}+4 \pi r^{3} P / c^{2}}{4 \pi r^{4} \bar{n}}\left(1-\frac{2 G M_{\mathrm{r}}}{r c^{2}}\right)^{-1 / 2}, \\
& \frac{\mathrm{d} P}{\mathrm{~d} a}=-\left(\rho+\frac{P}{c^{2}}\right) \frac{\mathrm{d} \Phi}{\mathrm{d} a},
\end{aligned}
$$

where $\bar{n}$ is the mean number density of baryons, $G$ is the Newtonian constant of gravitation and $c$ is the speed of light in vacuum. For any known temperature profile $T(a)$ inside the star, these equations are closed by an equation of state (EoS), which relates $\rho$ and $P$ to $\bar{n}$ and $T$. In the absence of a strong magnetic field, we neglect the dependence of $P$ and $\rho$ on $T$ (use a barotropic EoS) in the inner crust and the core, but take it into account in the outer crust and envelopes (Paper I).

The fifth equation relates the heat flux through a spherical surface to temperature gradient,

$L_{\mathrm{r}}=-\left(4 \pi r^{2}\right)^{2} \bar{n} \kappa \mathrm{e}^{-\Phi / c^{2}} \frac{\mathrm{de}^{\Phi / c^{2}} T}{\mathrm{~d} a}$,

where $\kappa$ is the thermal conductivity measured in the local reference frame. Finally, time-dependence is introduced by the equation (Richardson et al. 1979)

$\frac{\mathrm{d}\left(L_{\mathrm{r}} \mathrm{e}^{2 \Phi / c^{2}}\right)}{\mathrm{d} a}=\mathrm{e}^{2 \Phi / c^{2}}\left(\mathcal{E}-T \mathrm{e}^{-\Phi / c^{2}} \frac{\partial s}{\partial t}\right)$,

where $\mathcal{E}$ is the net rate of energy generation per baryon and $\partial s / \partial t$ is the coordinate time derivative of the entropy per baryon. The boundary condition for $\Phi$ is provided by the Schwarzschild metric outside the $\operatorname{star}(r>R)$,

$\mathrm{e}^{2 \Phi(R) / c^{2}}=1-2 G M / c^{2} R$,

where $R$ and $M=M_{\mathrm{R}}$ are the stellar radius and mass.

Equations (1)-(6) assume a spherically symmetric star in hydrostatic equilibrium. The dynamics of accreted matter is neglected, because in the case under study the accretion is extremely slow on the mechanical relaxation timescales of the concerned layers of the crust and core of the star.

Equation (6) can be combined with Eq. (5) to form

$\frac{c_{P}}{\bar{n}} \mathrm{e}^{\Phi / c^{2}} \frac{\partial T}{\partial t}=\frac{\partial}{\partial a}\left(4 \pi r^{2}\right)^{2} \bar{n} \kappa \mathrm{e}^{\Phi / c^{2}} \frac{\partial \tilde{T}}{\partial a}+\frac{\tilde{Q}}{\bar{n}}$,

where $c_{P}$ is the heat capacity per unit volume at constant pressure and

$\tilde{Q}=\tilde{Q}_{\mathrm{h}}-\tilde{Q}_{v}=\bar{n} \mathrm{e}^{2 \Phi / c^{2}} \mathcal{E}$, 
which is the net heating power density as seen by a distant observer. Here and hereafter we mark the quantities measured at infinity ("redshifted") by a tilde over their symbol. In Eqs. (8) and (9), $\tilde{T}=\mathrm{e}^{\Phi / c^{2}} T$ and $\tilde{Q}_{\mathrm{h}, v}=\mathrm{e}^{2 \Phi / c^{2}} Q_{\mathrm{h}, v}$, where $Q_{\mathrm{h}}$ and $Q_{v}$ are the local heating power and local neutrino emission power per unit volume, respectively. Assuming that the neutron star is fully transparent to neutrinos, one can calculate the total heat release or neutrino emission power in the frame of reference of a distant observer as (e.g., Yakovlev et al. 2003)

$\tilde{L}_{\mathrm{h}, v}=\int Q_{\mathrm{h}, v} \mathrm{e}^{2 \Phi / c^{2}} \mathrm{~d} V=4 \pi \int_{0}^{R} \frac{\mathrm{e}^{2 \Phi / c^{2}} Q_{\mathrm{h}, v} r^{2} \mathrm{~d} r}{\sqrt{1-2 G M_{\mathrm{r}} / r c^{2}}}$,

where $\mathrm{d} V$ is a proper volume element and the square root in the denominator is the volume correction factor (Thorne 1977).

Equation (8) can be written in the form of the usual thermal diffusion equation

$\frac{c_{P}}{\bar{n}} \frac{\partial \tilde{T}}{\partial t}=\frac{\partial}{\partial a}\left(4 \pi r^{2}\right)^{2} \bar{n} \kappa \mathrm{e}^{\Phi / c^{2}} \frac{\partial \tilde{T}}{\partial a}+\frac{\tilde{Q}}{\bar{n}}+\frac{c_{P}}{\bar{n}} \tilde{T} \frac{\partial \Phi}{c^{2} \partial t}$.

In practice, the last term on the right-hand side is much smaller than typical values of the left-hand side. In Paper I we treated it as an external source, with $\partial \Phi / \partial t$ evaluated from the solution at the preceding time step, but found it insignificant and therefore neglect it hereafter. The boundary condition to Eq. (11) at the stellar center is $\partial \tilde{T} / \partial a=0$. The outer boundary condition follows from Eq. (5) and reads

$$
\left.\frac{\partial \tilde{T}}{\partial a}\right|_{a=a_{\mathrm{b}}}=-\frac{\mathrm{e}^{\Phi / c^{2}} L_{\mathrm{b}}}{\left(4 \pi r^{2}\right)^{2} \bar{n} \kappa},
$$

where $L_{\mathrm{b}}$ is the energy flux through the outer boundary $a=a_{\mathrm{b}}$, which is provided by the quasi-stationary thermal structure of a thin envelope outside this boundary. We solve the nonstationary problem using the temperature-dependent EoS in the outer crust and choose the mass of the quasi-stationary envelope $\Delta M_{\mathrm{b}}$ so as to ensure that plasma is fully ionized at $\rho>\rho_{\mathrm{b}}$. In the absence of a strong magnetic field, this condition is guaranteed for $\Delta M_{\mathrm{b}}=$ $10^{-12} M_{\odot}$.

We solve the set of equations (1) - (12) by a finite-difference time-implicit scheme with an adaptive mesh and iterative refinements at each time-step, as described in Paper I.

\subsection{Equation of state and composition of the core and crust}

There are many theoretical approaches to construction of the EoS of superdense matter (see, e.g., the review by Oertel et al. 2017). For this work we have selected to use just two models, deemed to be the most representative.

The first model is BSk24 (Goriely et al. 2013; Pearson et al. 2019), which provides a unified treatment of the crust and the core of a neutron star, based on the same energy-density functional of a modified Skyrme type (so-called Brussels-Montreal functionals). This model is compatible with constraints derived from laboratory experiments, and in particular it ensures the highest accuracy of theoretically computed masses of atomic nuclei as compared to masses of thousands of different nuclear isotopes that have been measured in the laboratory. In the stellar core this EoS is consistent with the EoS of a neutron-star core calculated by Li \& Schulze (2008) within the BruecknerHartree-Fock approach, using the realistic Argonne V18 (Av18) nucleon-nucleon potential (Wiringa et al. 1995) and the phenomenological three-body forces that employ the same mesonexchange parameters as the Av18 potential.
The second EoS model is A18 $+\delta \mathrm{v}+\mathrm{UIX}^{*}$ (Akmal et al. 1998), named APR $^{*}$ for short. It is based on variational calculations using a two-body Av18 potential, supplemented by a modified three-body force UIX ${ }^{*}$ and so-called relativistic boost interaction (in computations we use the set of analytical fits to this EoS published in Appendix A of Paper I). The APR* EoS is applicable only to the core but not to the crust. In the nonaccreted crust, we supplement it by the SLy4 EoS (Douchin \& Haensel 2001; for analytic fits, see Haensel \& Potekhin 2004).

During accretion, the envelopes, ocean, and crust matter are gradually replaced by fresh material whose composition differs from the initial ground-state matter. In the outer envelopes, up to the density $\rho \sim 10^{8}-10^{9} \mathrm{~g} \mathrm{~cm}^{-3}$, the initial iron-group element composition is replaced by the material of the outer layers of the companion star or by the products of its thermonuclear burning (see Meisel et al. 2018 for review). For these accreted layers we adopt the layered structure model of Potekhin et al. (1997) with either $\mathrm{H}$ or $\mathrm{He}$ on the surface. As soon as the composition is known, all thermodynamic functions in the outer crust and the ocean are provided by the analytical model of a fully ionized Coulomb plasma (Potekhin \& Chabrier 2013).

Deeper in the crust, accreted matter is reprocessed by electron captures, neutron emissions, and pycnonuclear reactions. The reprocessed matter differs from the exact ground state, because for temperature $T \lesssim(4-5) \times 10^{9} \mathrm{~K}$ the nuclear reaction channels relevant for maintaining nuclear statistical equilibrium (photodissociation, photoabsorption) are closed (see e.g., Bisnovatyi-Kogan 2001 and references therein). In this case, practical models determining nuclear composition have been developed by Haensel \& Zdunik (1990, 2003, 2008). Furthermore, for $T \lesssim 3 \times 10^{9} \mathrm{~K}$, nuclear shell effects become important and further contribute to freeze the nuclear composition of the crust. The role of these effects in the formation of the accreted crust has recently been studied by Fantina et al. (2018). In the present study, we use and compare two of the most recent models (Haensel \& Zdunik 2008; Fantina et al. 2018), described below.

\subsection{Heat loss and production}

Cooling of an isolated neutron star goes through two major stages. The first, neutrino cooling stage lasts $\sim 10^{5}$ years. During this period, the core cools mostly via neutrino emission. The second, photon cooling stage begins when, with temperature decrease, the neutrino energy losses become smaller than the losses due to electromagnetic radiation from the surface. However, an accreting neutron star may become sufficiently hot again and return to the neutrino cooling regime.

Yakovlev et al. (2001) presented a comprehensive review of neutrino emission mechanisms in compact stars and supplied convenient fitting formulae for astrophysical applications. The most important reactions in the neutron-star crust and core with references to the appropriate fitting formulae are collected in Table 1 of Potekhin et al. (2015), which also includes references to several important updates in the relevant neutrino reaction rates, which improve the results of Yakovlev et al. (2001).

The most powerful neutrino emission occurs in the direct Urca process, but it operates only if the proton fraction $Y_{\mathrm{p}}$ exceeds some threshold value, $Y_{\mathrm{pDU}}$, which occurs above a certain threshold baryon number density $\bar{n}_{\mathrm{DU}}$. In the neutron-proton-electron (npe) matter $Y_{\mathrm{pDU}}=1 / 9$, but in the npe $\mu$ matter (with allowance for $\mu^{-}$-mesons) it is generally larger (e.g., Haensel 1995). The proton fraction as a function of the mean baryon number density $\bar{n}$ is uncertain, since it depends on the microscopic interaction model. A self-consistent modeling should employ the same 


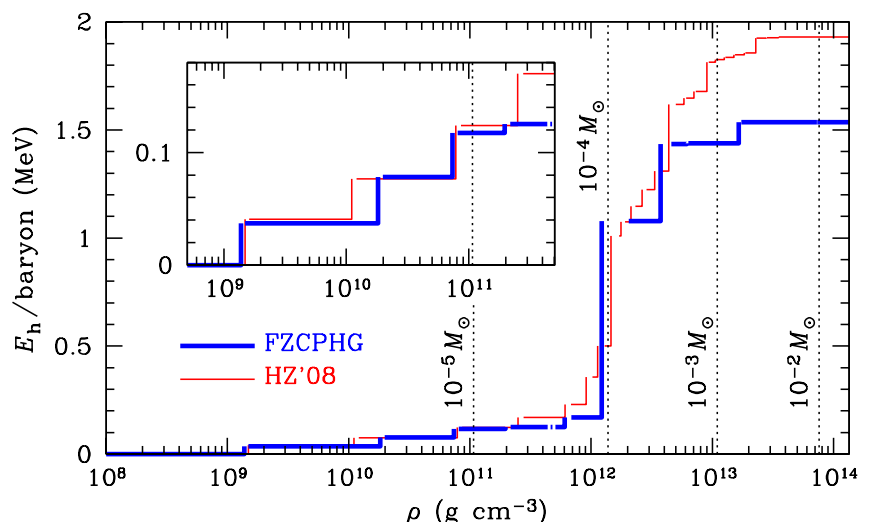

Fig. 1. Total heat $E_{\mathrm{h}}$ generated per accreted baryon as a function of mass density $\rho$, according to the models of Fantina et al. (2018; FZCPHG, thick blue line) and Haensel \& Zdunik (2008; HZ'08, thinner red line). The gaps in the lines correspond to the density discontinuities at the phase boundaries. The vertical dotted lines mark the $\rho$ values corresponding to four masses of accreted material, from $10^{-5} M_{\odot}$ to $10^{-2} M_{\odot}$, labeled near these lines, for a neutron star with gravitational mass $M=1.4 M_{\odot}$ and radius $R=12.6 \mathrm{~km}$. The inset shows a zoom to the low-density region.

interaction model for the EoS and for the proton fraction calculations. In this case, the threshold density depends on the EoS. Specifically, for the BSk24 and APR* models we have $\bar{n}_{\mathrm{DU}}=$ $0.0453 \mathrm{fm}^{-3}\left(Y_{\mathrm{pDU}}=0.136\right)$ and $\bar{n}_{\mathrm{DU}}=0.0783 \mathrm{fm}^{-3}\left(Y_{\mathrm{pDU}}=\right.$ $0.141)$, respectively. These densities are reached only in central parts of sufficiently massive neutron stars. The minimal mass of the star that allows the direct Urca processes to operate is $M_{\mathrm{DU}}=1.595 M_{\odot}$ for BSk24 and $M_{\mathrm{DU}}=2.01 M_{\odot}$ for $\mathrm{APR}^{*}$. In the absence of the direct Urca processes, the most important neutrino emission mechanisms in the core are the modified Urca (Murca) processes, baryon bremsstrahlung, and Cooper pair breaking and formation whenever the baryons are superfluid.

The nuclear transformations in the crust during accretion are accompanied with energy release. Part of this energy is lost to neutrino emission, but another part is transformed into heat which warms up the stellar crust. Here we consider two of the most recent models of the deep crustal heating, developed without and with allowance for the nuclear shell effects, respectively, by Haensel \& Zdunik (2008; HZ'08) and by Fantina et al. (2018; FZCPHG). There are several versions of each of these two models. For the first model (without nuclear shell effects), we choose the version of the accreted-crust composition and respective energy releases at the boundaries of different layers that is given in Table A. $3^{1}$ of Haensel \& Zdunik (2008). For the second one (with the shell effects) we adopt the results reported in Table A.1 of Fantina et al. (2018). Both tables correspond to the initial iron composition. The first table (HZ'08) is based on compressible liquid drop model by Mackie \& Baym (1977), while the second table (FZCPHG) corresponds to the BSk21 energy-density functional model (Goriely et al. 2010), which is similar to the BSk24 model that underlies the basic EoS used in the present work for the ground-state matter. The HZ'08 model predicts a total release of $1.93 \mathrm{MeV}$ of heat per accreted baryon, and the FZCPHG model predicts $1.54 \mathrm{MeV}$ per baryon. Figure 1 displays the total heat generated per accreted baryon, from the surface to a given density in the crust, as function of mass density. The vertical dotted lines

\footnotetext{
1 We have fixed a typo (" 0.8 " should read " 0.08 ") for the density discontinuity at $\rho=1.766 \times 10^{12} \mathrm{~g} \mathrm{~cm}^{-3}$.
}

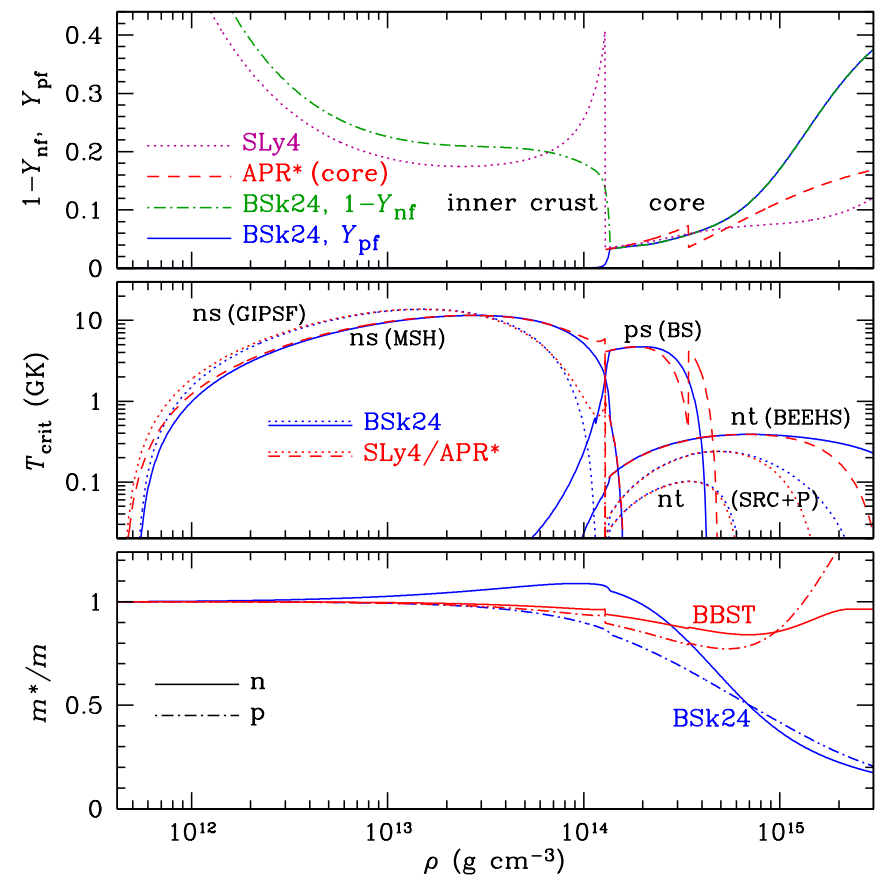

Fig. 2. Free nucleon fractions (top panel), critical temperatures (middle panel), and relative effective masses (bottom panel) for the basic theoretical models used in this paper for the nonaccreted crust and the core of a neutron star. The unified generalized Skyrme model BSk24 (Pearson et al. 2019) is compared with the variational APR* model for the core (Akmal et al. 1998), and with the SLy4 model (Douchin \& Haensel 2001) for the crust EoS and composition, as well as with the results of BBST for the effective masses. For the critical temperatures $T_{\text {crit }}$ of proton singlet, neutron singlet, and neutron triplet pairing types of superfluidity we employ the results of BS, MSH, and BEEHS, respectively, as parametrized by Ho et al. (2015). For comparison, by dotted lines in the middle panel we show the results of GIPSF for the neutron-singlet superfluidity in the neutron star crust, also parametrized by Ho et al. (2015), and the results of Ding et al. (2016) for the neutron-triplet superfluidity in the neutron star core, calculated with allowance for short-range correlations and polarization (SRC+P) for the effective potential models N3LO (upper dotted curves) and Av18 (lower dotted curves).

correspond to several masses of accreted material, from the surface to the given density, for a neutron star with gravitational mass $M=1.4 M_{\odot}$ and radius $R=12.6 \mathrm{~km}$, consistent with the BSk24 EoS.

\subsection{In-medium effects}

Neutrino emissivity of neutron stars can be strongly modified by in-medium (collective) effects, which affect the reaction rates in several ways (see Voskresensky 2001, for a review). Their simplest manifestation is the modifications of the effective nucleon masses $m_{\mathrm{p}}^{*}$ and $m_{\mathrm{n}}^{*}$ owing to distortion of the dispersion relation. The values of these effective masses should be taken from microscopic theories. The ratios $m^{*} / m$ affect not only the neutrino emission rates, but also baryon thermal conductivities, and thus they have a complex effect on thermal evolution of the star. The ratios $m_{\mathrm{n}}^{*} / m_{\mathrm{n}}$ and $m_{\mathrm{p}}^{*} / m_{\mathrm{p}}$ (respectively, for neutrons and protons) used in the present work are plotted in the bottom panel of Fig. 2 as functions of the mean baryon density $\bar{n}$, according to the microscopic theories consistent with the two equations of state that we employ in this work. For BSk24, they are given by Eq. (A.10) of Chamel et al. (2009) with the parameters 
listed in Goriely et al. (2013). For APR*, we use Eq. (6) of Baldo et al. (2014, hereafter BBST) with the parameters for the effective two- and three-body potentials Av18+UIX that underlie the EoS APR*. The number densities of the free nucleons of each type, which are needed in these equations, are calculated according to the fitting formulas given in Pearson et al. (2019) for BSk24 and in Paper I for APR*.

However, the effective mass approximation may be insufficient, as it is unable to describe some qualitative in-medium effects that are absent in the free space. The nucleon correlations also affect reaction matrix elements and propagators and modify the density of intermediate states. In particular, according to Schaab et al. (1997), the in-medium effects enhance emissivity in the Murca process and result in a strong density dependence, which gives a smooth crossover from the standard to the enhanced cooling scenario for increasing star masses. A qualitatively similar effect has been found by Shternin et al. (2018), who described the in-medium nucleon scattering in the Brueckner-Hartree-Fock approximation taking into account the effective two- and three-body forces and the Pauli blocking of intermediate states. These authors suggested a simple expression for the medium-enhanced emissivity of the neutron branch of the Murca process, which has been incorporated in our code. The importance of this enhancement of the Murca process for neutron star cooling has been demonstrated by Shternin et al. (2018) as well as in Paper I.

\subsection{Baryon superfluidity}

Baryon superfluidity is known to affect the thermal evolution of neutron stars, first due to its influence on the heat capacity, neutrino emissivity, and heat transport, and second due to the emergence of a specific neutrino emission mechanism by Cooper pair breaking and formation (PBF; see, e.g., the reviews by Page et al. 2013 and by Schmitt \& Shternin 2018). The PBF processes are most powerful at $T \sim T_{\text {crit }}$, where $T_{\text {crit }}$ is a critical temperature specific to each type of superfluidity (e.g., Leinson 2010, and references therein). Microscopic theories and methods that are being employed to understand the basic properties of superfluid nuclear systems, with emphasis on the matter of neutron stars, have recently been reviewed by Sedrakian \& Clark (2018).

To incorporate these effects into astrophysical modeling, we use the convenient fitting formulas collected by Yakovlev et al. (2001) with updates and corrections listed in Potekhin et al. (2015). As a rule, these fitting formulas describe the effects of superfluidity as functions of $T / T_{\text {crit }}$, where the critical temperature $T_{\text {crit }}$ depends on the nucleon type (neutrons or protons) and their Cooper pairing type. For each type of superfluidity, $T_{\text {crit }}$ also depends on the number density of free nucleons. Different theoretical results for these dependences have been parametrized by Ho et al. (2015). In the present work, we consider the parametrizations that describe theoretical results of Baldo \& Schulze (2007; BS) for proton singlet $\left({ }^{1} S_{0}\right)$ pairing type, Baldo et al. (1998; BEEHS) for neutron triplet $\left({ }^{3} P_{2}-\right.$ ${ }^{3} F_{2}$ ) pairing type, and either Margueron et al. (2008; $\left.\mathrm{MSH}\right)$ or Gandolfi et al. (2009; GIPSF) for neutron singlet $\left({ }^{1} S_{0}\right)$ pairing type. The first two types of superfluidity are most relevant in the core of a neutron star, and the last one in the crust.

The top panel of Fig. 2 shows number fractions of free neutrons $\left(Y_{\mathrm{nf}}\right)$ and protons $\left(Y_{\mathrm{pf}}\right)$ as functions of the mean baryon number density $\bar{n}$ in the inner crust and in the core of a neutron star for the EoS models described above. Corresponding values of $T_{\text {crit }}$ as functions of $\bar{n}$ are shown in the middle panel for the above-mentioned theoretical models of baryon pairing gaps.
Recent studies have demonstrated that the effects of manybody correlations on baryon superfluidity can suppress the superfluid gap, and consequently $T_{\text {crit }}$, by an order of magnitude or even stronger for the triplet type of pairing ${ }^{3} P_{2}-{ }^{3} F_{2}$ (e.g., Ding et al. 2016; see Sedrakian \& Clark 2018 for a discussion). The suppressed critical temperatures are also shown in the middle panel of Fig. 2 (below the BEEHS curves). The influence of this effect on the cooling of isolated neutron stars has recently been studied by Wei et al. (2019). In Sect. 5 we test its influence on the quiescent thermal states of neutron stars in the SXTs.

\section{Equilibrium thermal luminosities in quiescence}

\subsection{Observations}

During a long period covering many outbursts, the interior of a neutron star in an SXT becomes appreciably heated by the part of the deep crustal heat that flows into the core during accretion. The temperature of the core $T_{\text {core }}$ thus increases until this heating is balanced by the neutrino energy loss. The higher the average mass accretion rate $\langle\dot{M}\rangle$, the higher the equilibrium $T_{\text {core }}$ value at the crust-core boundary. This value determines a thermal equilibrium state that the crust tends to acquire in quiescence. Thus, thermal photon luminosity of the SXT in quiescence, $L_{\mathrm{q}}$, is correlated with $\langle\dot{M}\rangle$. A concrete value of $L_{\mathrm{q}}$ at a given $\langle\dot{M}\rangle$ depends on the neutron star parameters and on the properties of the dense matter in the interior of the star. It also depends on the properties of the heat blanketing envelopes: if the accreted matter has been burnt to heavy chemical elements (one usually takes iron for a fiducial model), the thermal luminosity is lower than in the cases where the heat transport is controlled by layers composed of relatively light chemical elements, for instance if the mass of residual helium is sufficiently large to fill the heat blanket (see, e.g., Yakovlev et al. 2004, and references therein). Therefore, the simultaneous consideration of $\langle\dot{M}\rangle$ and $L_{\mathrm{q}}$ can help to determine neutron-star parameters and probe the properties of the interior of a neutron star.

Yakovlev et al. (2003) were the first to undertake such a study. They considered five SXTs whose average accretion rate and quiescent luminosity had been estimated by that time. More comprehensive compilations of the properties of the SXTs in quiescence were published by Heinke et al. (2007, 2009, 2010). Thereafter, these data, for 24 SXTs in total, have been traditionally quoted in different reviews and research papers (e.g., Wijnands et al. 2013, 2017; Beznogov \& Yakovlev 2015a,b) for analysis of the $L_{\mathrm{q}}(\langle\dot{M}\rangle)$ correlations. Some sources beyond this sample have been discussed in the context of such an analysis from time to time (e.g., 1RXS J180408.9-342058 has been added to the sample by Parikh et al. 2018, and SAX J1750.8-2900 was discussed by Lowell et al. 2012 and by Parikh \& Wijnands 2017 and plotted in a figure by Fortin et al. 2018), and the average mass transfer rates for some of the SXTs have been revisited (Coriat et al. 2012; Heinke et al. 2013; Van et al. 2019), but a systematic revision of the cumulative dataset has not been undertaken.

Here we present a renewed and more comprehensive compilation of the observational data pertinent to the analysis of the quasi-equilibrium thermal states of neutron stars in the SXPs in quiescence. Tables 1 and 2 present 35 objects that are suitable for the analysis of the $L_{\mathrm{q}}(\langle\dot{M}\rangle)$ dependence. We have not only expanded the list, but have also updated the average accretion rates and/or quiescent luminosities for most of the 24 previously tabulated SXTs. In order to preserve continuity with the previous works, the SXTs numbered 1-15 in our tables are the same objects as in Heinke et al. (2010) and Wijnands et al. (2017), 
Table 1. SXTs with estimated average accretion rates and quiescent thermal luminosities.

\begin{tabular}{|c|c|c|}
\hline No. & Source & $\operatorname{Remark}^{(a)}$ \\
\hline 1 & 4U 2129+47 (V* V1727 Cyg) & In NGC 7078 \\
\hline 2 & KS $1731-260$ & $\mathrm{CC}, \mathrm{QP}$ \\
\hline 3 & $4 \mathrm{U} 1608-522$ & \\
\hline 4 & EXO 1745-248 (Ter 5 X-1) & In Terzan 5 \\
\hline 5 & $1 \mathrm{M} 1716-315(1 \mathrm{H} 1715-321)$ & \\
\hline \multirow[t]{2}{*}{6} & RX J1709.5-2639 & \\
\hline & (XTE J1709-267) & In NGC 6293 \\
\hline 7 & MXB 1659-29 (XB 1658-298) & $\mathrm{CC}, \mathrm{QP}, \mathrm{E}$ \\
\hline \multirow[t]{2}{*}{8} & 1RXS J173546.9-302859 & \\
\hline & $(\mathrm{XB} 1732-304)$ & In Terzan 1 \\
\hline 9 & 4U 1456-32 (Cen X-4) & \\
\hline 10 & $1 \mathrm{H} 1905+00(4 \mathrm{U} 1857+01)$ & \\
\hline 11 & SAX J1806.8-2435 (2S 1803-245) & \\
\hline 12 & 4U 1730-22 & \\
\hline 13 & EXO 1747-214 & \\
\hline 14 & XTE $2123-058$ & \\
\hline 15 & SAX J1810.8-2609 & \\
\hline 16 & $4 \mathrm{U} 1908+005(\mathrm{Aql} \mathrm{X}-1)$ & $\mathrm{CC}$ \\
\hline 17 & SAX J1748.9-2021 (NGC 6440 X-1) & In NGC 6440 \\
\hline \multirow[t]{2}{*}{18} & CXOGlb J174852.7-202124 & UC \\
\hline & $($ NGC 6440 X-2) & in NGC 6440 \\
\hline 19 & XTE J0929-314 (V* BW Ant) & $\mathrm{UC}$ \\
\hline 20 & SAX J1808.4-3658 (V* V4580 Sgr) & \\
\hline 21 & XTE J1807-294 & $\mathrm{UC}$ \\
\hline 22 & XTE J1751-305 & $\mathrm{UC}$ \\
\hline 23 & XTE J1814-338 (V* V5511 Sgr) & \\
\hline 24 & IGR J00291+5934 (V* V1037 Cas) & \\
\hline 25 & HETE J1900.1-2455 & $\mathrm{CC}, \mathrm{QP}$ \\
\hline 26 & XTE J1701-462 & $\mathrm{CC}, \mathrm{QP}$ \\
\hline \multirow[t]{2}{*}{27} & IGR J17480-2446 & $\mathrm{CC}$ \\
\hline & $(\operatorname{Ter} 5 \mathrm{X}-2)$ & in Terzan 5 \\
\hline 28 & EXO 0748-676 (V* UY Vol) & $\mathrm{CC}, \mathrm{QP}, \mathrm{E}$ \\
\hline 29 & 1RXS J180408.9-342058 & $\mathrm{CC}$ \\
\hline \multirow[t]{2}{*}{30} & Swift J174805.3-244637 & $\mathrm{CC}$ \\
\hline & $($ Ter $5 X-3)$ & in Terzan 5 \\
\hline 31 & SAX J1750.8-2900 & \\
\hline 32 & Swift J1756.9-2508 & $\mathrm{UC}$ \\
\hline \multirow[t]{2}{*}{33} & Swift J1750.7-3117 & $\mathrm{E}$ \\
\hline & (GRS 1747-312) & in Terzan 6 \\
\hline 34 & IGR J18245-2452 & In Messier 28 \\
\hline 35 & MAXI J0556-332 & $\mathrm{CC}, \mathrm{QP}$ \\
\hline
\end{tabular}

Notes. The first column gives the sequential number for a quick reference; the second column lists the most common source identifiers in the literature; and the last column indicates a particular source type or association. ${ }^{(a)} \mathrm{UC}$ - ultra-compact source (Van et al. 2019), E - eclipser, $\mathrm{QP}$ - quasi-persistent source, CC - crust cooling source (Wijnands et al. 2017).

while our SXT numbers 16 through 24 have been previously labeled by letters A through I, respectively.

It should be noted that the accretion rates are usually evaluated from observed X-ray luminosities $\tilde{L}_{\mathrm{X}}$ using the equation (e.g., Van et al. 2019)

$\dot{M}_{\mathrm{obs}}=\frac{\tilde{L}_{\mathrm{X}} R_{\mathrm{f}}}{G M_{\mathrm{f}}}$

where $M_{\mathrm{f}}$ and $R_{\mathrm{f}}$ are the fiducial mass and radius of the neutron star. In most of the previous works, the "canonical neutron star model" with $M_{\mathrm{f}}=1.4 M_{\odot}$ and $R_{\mathrm{f}}=10 \mathrm{~km}$ was used for calculation of $\left\langle\dot{M}_{\text {obs }}\right\rangle$ (e.g., Degenaar \& Wijnands 2012), which is sometimes written as $\tilde{L}_{\mathrm{X}} \approx 0.2 \dot{M}_{\mathrm{obs}} c^{2}$. Van et al. (2019) derived $\dot{M}_{\text {obs }}$ from $\tilde{L}_{\mathrm{X}}$ assuming $M_{\mathrm{f}}=1.4 M_{\odot}$ and $R_{\mathrm{f}}=11.5 \mathrm{~km}$. Although the latter radius is more realistic, we have rescaled the corresponding values of $\left\langle\dot{M}_{\text {obs }}\right\rangle$ in Table 2 (lines 17, 18, 21, 22, 25, 32, and 34 ) back to the canonical model for the uniformity of the data sample.

The total (bolometric) accretion luminosity measured at infinity is related to the accretion rate $\dot{M}$ measured locally at the neutron star surface by equation (e.g., Mitra 1998; Meisel et al. 2018)

$\tilde{L}_{A}=\frac{z}{(1+z)^{2}} \dot{M} c^{2}$

where

$z=\left(1-2 G M / R c^{2}\right)^{-1 / 2}-1$,

which is the gravitational redshift at the stellar surface and $\tilde{L}_{A}=$ $A \tilde{L}_{\mathrm{X}}, A>1$ being the bolometric correction. Excluding $\tilde{L}_{\mathrm{X}}$ from Eqs. (13) and (14), we obtain

$\dot{M}_{\mathrm{obs}}=\frac{z}{z_{\mathrm{f}}}\left(\frac{1+z_{\mathrm{f}}}{1+z}\right)^{2} \frac{\dot{M} / A}{1+z_{\mathrm{f}} / 2}$,

where $z_{\mathrm{f}}$ is the fiducial gravitational redshift given by Eq. (15) with $M=M_{\mathrm{f}}$ and $R=R_{\mathrm{f}}\left(z_{\mathrm{f}}=0.3057\right.$ for the canonical neutron star model).

Most of the values in Table 2 are taken from papers indicated by the numbers in square brackets. The cases where the listed values are not plainly adopted, but are derived in this work from the given references, are marked by a footnote to the table. In particular, whenever the uncertainties of bolometric luminosities are not given explicitly, we evaluate them from the uncertainties of effective temperatures and/or the scattering of results obtained with using different spectral models. For sources with just one observed outburst, the average mass-accretion rate is reported in Table 2 as an upper limit, because the duration of the quiescent period can be much larger than the X-ray observation time-line $(\sim 30 \mathrm{yr})^{2}$. We note that in many cases there can be much larger systematic uncertainties due to unaccounted model-dependence, poorly known distance, or hydrogen column density, and so on, therefore the listed errors should be considered as lower limits to largely unknown actual uncertainties.

Some of the considered SXTs or listed numbers warrant the following additional comments.

1. $4 U 2129+47$. The bolometric luminosity is derived from the effective temperature and radius obtained by Nowak et al. (2002) for the canonical neutron star model (spectral fit models $\mathrm{E}$ and $\mathrm{F}$ in Table 2 of that paper), and its uncertainties are roughly

2 According to a disk instability model, the maximal duration of the quiescence period is $\sim 180 \mathrm{yr}$ (see, e.g., Sect. 6.4 in Lasota 2001). Estimates by Chugunov et al. (2014) suggest that an even longer quiescence period ( $1000 \mathrm{yr}$ ) should be allowed, if all X-ray sources known as candidate quiescent LMXBs in globular clusters are indeed LMXBs in quiescent state. These candidate sources are selected by X-ray spectrum, which is well fitted by neutron star thermal emission, but they are treated as candidates because no outburst from these source has been detected yet (see, e.g., Bahramian et al. 2015 and references therein). An alternative explanation of these sources, suggested by Chugunov et al. (2014), is based on heating associated with Chandrasekhar-FriedmanSchutz instability (Friedman \& Schutz 1978a,b) and does not require such a long quiescence time in LMXBs. 
A. Y. Potekhin et al.: Thermal evolution of soft X-ray transients

Table 2. Key properties of SXTs with estimated average accretion rates and quiescent thermal luminosities.

\begin{tabular}{|c|c|c|c|c|c|c|c|}
\hline No. & Short name & $\begin{array}{c}\left\langle\dot{M}_{\mathrm{obs}}\right\rangle \\
\left(M_{\odot} \mathrm{yr}^{-1}\right)\end{array}$ & $\begin{array}{c}\tilde{L}_{\mathrm{q}} \\
\left(10^{33} \mathrm{erg} \mathrm{s}^{-1}\right)\end{array}$ & $\begin{array}{l}P_{\text {orb }} \\
\text { (h) }\end{array}$ & $\begin{array}{l}\text { Spin } \\
(\mathrm{Hz})\end{array}$ & $\begin{array}{c}M_{\mathrm{d}} \\
\left(M_{\odot}\right)\end{array}$ & $\begin{array}{c}\text { Distance } \\
(\mathrm{kpc})\end{array}$ \\
\hline 1 & $4 U$ 2129+47 & $3.9 \times 10^{-9}[1]$ & $1.5_{-11}^{+3.1}[2,3]^{(a)}$ & $5.96[1]$ & - & - & $10.3 \pm 0.4[4]$ \\
\hline 2 & KS $1731-260$ & $<9 \times 10^{-10}[5]{ }^{(b)}$ & $0.39 \pm 0.03[6]$ & $>2[7]$ & $524[8]$ & - & $7.2 \pm 1.0[9]$ \\
\hline 3 & $4 U 1608-522$ & $9.6 \times 10^{-10}$ & $5.3_{-2.9}^{+4.7}[10,11]{ }^{(a)}$ & $10-125[7]$ & $620[8]$ & - & $4.1 \pm 0.4[9]$ \\
\hline 4 & Ter 5 X-1 & $3 \times 10^{-11}[12]$ & $<0.1[12,13]$ & - & 333 [14] & - & $5.5 \pm 0.9[15]$ \\
\hline 5 & $1 \mathrm{M} 1716-315$ & $<2.5 \times 10^{-10}[2]$ & $1.3_{-07}^{+1.2}[2,16]{ }^{(a)}$ & - & - & - & $5.1-6.9[17]$ \\
\hline 6 & XTE J1709 & $1.8 \times 10^{-10}[10]$ & $1.4_{-0.5}^{+0.6}[18]^{(b)}$ & - & - & - & $8.5-8.8[18,19]$ \\
\hline 7 & MXB 1659-29 & $1.4 \times 10^{-10}[10]$ & $0.20_{-0.11}^{+0.05}[10,20]{ }^{(a)}$ & $7.11[9]$ & $567[8]$ & $0.3-0.8[21]$ & $12 \pm 3[9]$ \\
\hline 8 & XB 1732-304 & $<1.5 \times 10^{-10}[10]$ & $<1.1[10]$ & - & - & - & $5.2 \pm 0.5[22]$ \\
\hline 9 & Cen X-4 & $3.8 \times 10^{-11}[1]$ & $0.12 \pm 0.01[23]^{(b)}$ & $15.1[24]$ & - & $0.31 \pm 0.27[25]^{(c)}$ & $1.2 \pm 0.2[1]$ \\
\hline 10 & $1 \mathrm{H} 1905+00$ & $<1.1 \times 10^{-10}[2]$ & $<0.01$ & $<1.5[2]$ & - & - & $10[26]^{(d)}$ \\
\hline 11 & $2 S 1803-245$ & $<7 \times 10^{-11}[2]$ & $<0.52[2]$ & $\sim 9$ [2] & - & - & $7.3[2,27]{ }^{(d)}$ \\
\hline 12 & 4U 1730-22 & $<4.8 \times 10^{-11}[2]$ & $2.2_{-1.1}^{+2.0}[2,28](a)$ & - & - & - & $10_{-4}^{+12}[28]$ \\
\hline 13 & EXO 1747 & $<3 \times 10^{-11}[10]$ & $<0.07[10]$ & - & - & - & $11[9,29]{ }^{(d)}$ \\
\hline 14 & XTE 2123 & $<7 \times 10^{-12}[1]$ & $<0.14[10]$ & $5.956[7]$ & - & $0.76 \pm 0.22[30]^{(e)}$ & $9.6 \pm 1.3[30]$ \\
\hline 15 & SAX J1810.8 & $5 \times 10^{-12}$ & $<0.2[10]$ & - & $532[32]$ & - & $4.9 \pm 0.3[33]$ \\
\hline 16 & Aql X-1 & $3.2 \times 10^{-10}[34]{ }^{(b)}$ & $2.1 \pm 0.5[34]^{(b)}$ & $18.9[7]$ & $550[7]$ & - & $3.0-6.1[7]$ \\
\hline 17 & NGC 6440 X-1 & $6 \times 10^{-11}$ & $1.3 \pm 0.4[36]^{(b)}$ & 8.765 [37] & 442 [37] & $0.12-1[37]$ & $8.5 \pm 0.6$ \\
\hline 18 & NGC 6440 X-2 & $8.4 \times 10^{-13}[35]$ & $<0.023[38]^{(b)}$ & 0.960 [39] & 206 [39] & $\sim 0.0076[39]$ & $8.5 \pm 0.4[40]$ \\
\hline 19 & XTE J0929 & $\lesssim 2 \times 10^{-11}[2]$ & $<0.1[2]{ }^{(f)}$ & $0.726[41]$ & 185 [41] & $\sim 0.01[41]$ & $8_{-3}^{+7}[40]$ \\
\hline 20 & SAX J1808.4 & $1.7 \times 10^{-11}$ & $<0.02_{[2]}^{(f)}$ & $2.014[42]$ & $401[42]$ & $0.04_{-0.01}^{+0.02}[43]$ & $3.4-3.6[1]$ \\
\hline 21 & XTE J1807 & $\lesssim 3 \times 10^{-11}{ }_{[35]}^{(g)}$ & $<0.13[2]$ & $0.667[44]$ & $190.6[44]$ & - & $4.4 \pm 0.6[44]$ \\
\hline 22 & XTE J1751 & $4.3 \times 10^{-12}[35]$ & $<0.4[2]$ & $0.707[45]$ & $245[8]$ & $0.014-0.035$ [45] & $8_{-1.3}^{+0.5}[40]$ \\
\hline 23 & XTE J1814 & $6 \times 10^{-12}$ & $<0.17$ [2] & $4.275[7]$ & 314 [7] & $0.19-0.32[46]^{(h)}$ & $8.0 \pm 1.6[7]$ \\
\hline 24 & IGR J00291 & $\sim 2.2 \times 10^{-12}{ }_{[47]}^{(c)}$ & $0.19_{-0.08}^{+0.06}[2]$ & $2.46[48]$ & $599[48]$ & $0.039-0.16[48]$ & $2.6-3.6[1]$ \\
\hline 25 & HETE J1900.1 & $3.9 \times 10^{-11}[35]$ & $0.061 \pm 0.037[49]$ & $1.388[50]$ & 377 [50] & $0.016-0.07[50]$ & $4.7 \pm 0.6[7]$ \\
\hline 26 & XTE J1701 & $<9 \times 10^{-10}{ }_{[51]}^{(b)}$ & $<5[51,52]{ }^{(f)}$ & - & - & - & $7.3-8.8[53]$ \\
\hline 27 & Ter $5 \mathrm{X}-2$ & $<1.7 \times 10^{-11}[54](f)$ & $0.7 \pm 0.1$ & $21.274[55]$ & $11.04[55]$ & $\gtrsim 0.4$ [55] & $5.5 \pm 0.9[15]$ \\
\hline 28 & EXO 0748 & $<4.4 \times 10^{-10}$ & $3.8 \pm 0.2[56]$ & $3.824[57]$ & $552[8]$ & $\sim 0.1[58]$ & $7.4 \pm 0.9[9]$ \\
\hline 29 & 1RXS J180408 & $<4.6 \times 10^{-11}$ & $0.74_{-0.18}^{+0.09}{ }^{[59]}{ }^{(b)}$ & - & - & - & $5.8[59]{ }^{(d)}$ \\
\hline 30 & Ter 5 X-3 & $<3 \times 10^{-11}{ }_{[60]}(f)$ & $1.2 \pm 0.2[61]^{(i)}$ & - & - & - & $5.5 \pm 0.9[15]$ \\
\hline 31 & SAX J1750.8 & $2 \times 10^{-10}[62]$ & $<2.8[63]$ & - & $601[8]$ & - & $6.79 \pm 0.14$ \\
\hline 32 & Swift J1756.9 & $1.5 \times 10^{-11}[35]$ & $<1.0[38]^{(b)}$ & $0.912[64]$ & 182 [64] & $0.007-0.03[64]$ & $8 \pm 4$ \\
\hline 33 & GRS 1747 & $1.0 \times 10^{-10}[65]$ & $<2.4[65]$ & $12.360[66]$ & - & - & $9.5_{-2.5}^{+3.3}[4]$ \\
\hline 34 & IGR J18245 & $\lesssim 10^{-10}[35]$ & $<0.07[67]$ & $11.026[68]$ & 254 [68] & $\sim 0.2[68]$ & $5.5_{-0.4}^{+0.2}[68,69]$ \\
\hline 35 & MAXI J0556 & $<10^{-9}{ }_{[70]}(f)$ & $<5.1[71](f)$ & {$[72]^{(j)}$} & - & $0.45[72]$ & $43.6_{-1.6}^{+0.9}[71]$ \\
\hline
\end{tabular}

Notes. Each row gives the sequential number; source name (may be truncated; see Table 1 for the full identifiers); estimate of the long-term averaged mass accretion rate; observed thermal luminosity in quiescence; orbital period; estimate of the companion (donor star) mass (some mass ratio estimates are given in the footnotes); distance estimate. ${ }^{(a)}$ The statistical errors are evaluated based on the data in the second reference. ${ }^{(b)}$ Estimated in this work, using data from the given reference. ${ }^{(c)}$ Donor/accretor mass ratio $M_{\mathrm{d}} / M_{\mathrm{a}}=0.18 \pm 0.06$ [12]. ${ }^{(d)} \mathrm{An}$ upper limit and an assumed distance. ${ }^{(e)}$ Donor/accretor mass ratio $M_{\mathrm{d}} / M_{\mathrm{a}}=0.49 \pm 0.10$ [13]. ${ }^{(f)}$ Conservative upper limit, based on data from the given reference. ${ }^{(g)}$ Estimated upper bound, including the uncertainty given in this reference. ${ }^{(h)}$ Donor/accretor mass ratio $M_{\mathrm{d}} / M_{\mathrm{a}}=0.123_{-0.01}^{+0.012}[34] .{ }^{(i)}$ The coldest measurement, which agrees with the pre-outburst level. ${ }^{(j)}$ Two candidate solutions, $16.4 \mathrm{~h}$ and $9.75 \mathrm{~h}$.

References. [1] Coriat et al. (2012); [2] Heinke et al. (2009); [3] Nowak et al. (2002); [4] Kuulkers et al. (2003); [5] Ootes et al. (2016); [6] Merritt et al. (2016); [7] Watts et al. (2008); [8] Watts (2012); [9] Galloway et al. (2008); [10] Heinke et al. (2007); [11] Rutledge et al. (1999); [12] Degenaar \& Wijnands (2012); [13] Rivera Sandoval et al. (2018); [14] Matrange et al. (2017); [15] Ortolani et al. (2007); [16] Jonker et al. (2007a); [17] Jonker \& Nelemans (2004); [18] Degenaar et al. (2013); [19] Jonker et al. (2004); [20] Cackett et al. (2013); [21] Ponti et al. (2018); [22] Ortolani et al. (1999); [23] Cackett et al. (2010); [24] Cowley et al. (1988); [25] D' Avanzo et al. (2005); [26] Jonker et al. (2007b); [27] Cornelisse et al. (2007); [28] Tomsick et al. (2007); [29] Tomsick et al. (2005); [30] Casares et al. (2002); [31] Fiocchi et al. (2009); [32] Bilous et al. (2018); [33] Allen et al. (2018); [34] Ootes et al. (2018); [35] Van et al. (2019), accretion rate is rescaled to the canonical neutron star; [36] Walsh et al. (2015); [37] Sanna et al. (2016); [38] Haskell et al. (2012); [39] Bult et al. (2015); [40] Heinke et al. (2013); [41] Galloway et al. (2002); [42] Chakrabarty \& Morgan (1998); [43] Wang et al. (2013); [44] Riggio et al. (2008); [45] Markwardt et al. (2002); [46] Wang et al. (2017); [47] De Falco et al. (2017); [48] Galloway et al. (2005); [49] Degenaar et al. (2017); [50] Kaaret et al. (2006); [51] Turlione et al. (2015); [52] Fridriksson et al. (2011); [53] Lin et al. (2009) [54] Ootes et al. (2019); [55] Papitto et al. (2011); [56] Degenaar et al. (2014) [57] Parmar et al. (1986); [58] Mikles \& Hynes (2012); [59] Parikh et al. (2018); [60] Bahramian et al. (2014); [61] Degenaar et al. (2015); [62] Lowell et al. (2012); [63] Parikh \& Wijnands (2017); [64] Krimm et al. (2009); [65] Vats et al. (2018); [66] in 't Zand et al. (2003); [67] Linares et al. (2014); [68] Papitto et al. (2013); [69] Becker et al. (2003); [70] Homan et al. (2014); [71] Parikh et al. (2017); [72] Cornelisse et al. (2012). 
estimated from the given temperature uncertainties and scattering of different estimates in that publication. The same value of $\tilde{L}_{\mathrm{q}}$ has been given by Heinke et al. (2009).

2. KS 1731-260. The average accretion rate is estimated as the average rate during the outburst $\left(1.5 \times 10^{17} \mathrm{~g} \mathrm{~s}^{-1}\right.$, Ootes et al. 2016) multiplied by the observed outburst duration (11.5 years) and divided by the total time of observations ( $\approx 30$ years). This estimate is very uncertain because only one transition between the active and quiescent states (the end of the outburst in 2001) has been observed. We derive the statistical errors on $\tilde{L}_{\mathrm{q}}$ from the errors on the effective temperature given by Merritt et al. (2016), however the authors warn that there can be large systematic errors.

4. EXO 1745-248 (Terzan $5 X-1)$. The luminosity is completely dominated by nonthermal emission (e.g., Degenaar \& Wijnands 2012). The limit $\tilde{L}_{\mathrm{q}}<2.1 \times 10^{33} \mathrm{erg} \mathrm{s}^{-1}$ was most often quoted (e.g., Heinke et al. 2007; Beznogov \& Yakovlev 2015a). Rivera Sandoval et al. (2018) found a strong variability of X-ray luminosity of this SXT in quiescence, with a luminosity variation in the $0.5-10 \mathrm{keV}$ energy range from $3 \times 10^{31} \mathrm{erg} \mathrm{s}^{-1}$ to $2 \times 10^{34} \mathrm{erg} \mathrm{s}^{-1}$. Since the total thermal and nonthermal luminosity cannot be smaller than the quasi-equilibrium thermal component, we adopt $3 \times 10^{31} \mathrm{erg} \mathrm{s}^{-1}$ as an upper limit to this component in the X-rays. For the bolometric quiescent luminosity, this implies the conservative upper limit $\tilde{L}_{\mathrm{q}}<10^{32} \mathrm{erg} \mathrm{s}^{-1}$, which is consistent, within uncertainties, with the constraint $\tilde{L}_{\mathrm{q}} \lesssim 7 \times 10^{31} \mathrm{erg} \mathrm{s}^{-1}$ obtained by Degenaar \& Wijnands (2012) in frames of a specific spectral model.

7. MXB 1659-29. This bolometric luminosity corresponds to the effective temperature estimate $k \tilde{T}_{\text {eff }}=55 \pm 3 \mathrm{eV}$ obtained by Cackett et al. (2013) for observations performed $11 \mathrm{yr}$ after the end of the outburst. This estimate is consistent with the previous two, for observations taken approximately 4 and 7 years earlier (Cackett et al. 2005, 2008). However, the count rate has dropped by a factor of three in the latest observation compared with the previous two (possibly due to an increase in hydrogen column density; see discussion in Cackett et al. 2013). Inclusion of a power-law component improves the spectral fit and gives $k \tilde{T}_{\text {eff }} \approx 45 \mathrm{eV}\left(\tilde{L}_{\mathrm{q}} \sim 7 \times 10^{31} \mathrm{erg} \mathrm{s}^{-1}\right)$, which is reflected in the larger negative error estimate in our table, but the powerlaw component is not required to fit the previous observations (Cackett et al. 2013).

9. Cen X-4. Luminosity estimate is based on the temperature reported in Table 2 of Cackett et al. (2010) for Suzaku observation (the latest and the coldest one) and the canonical neutron star parameters.

16. $4 U 1908+005$ (Aql $X-1)$. The long-term average accretion rate is calculated as the total accreted mass during the period of regular observations from 1996 to 2015, determined from Table 2 of Ootes et al. (2018), divided by this time interval. We note that this rate was $\sim 40 \%$ higher in the first five years of this period. Because of frequent outbursts, this neutron star never reaches thermal equilibrium (Ootes et al. 2018). Here, the baseline quiescent luminosity at infinity $\tilde{L}_{\mathrm{q}}$ is calculated from the range of base levels of the effective temperature in the numerical fitting simulations by Ootes et al. (2018), for the adopted values of $M=1.6 M_{\odot}$ and $R=11 \mathrm{~km}$.

19. XTE J0929-314. This limit on $\left\langle\dot{M}_{\text {obs }}\right\rangle$ roughly agrees with the refined constraint $\left\langle\dot{M}_{\text {obs }}\right\rangle<\left(8.4_{-6.7}^{+22}\right) \times 10^{-12} M_{\odot} \mathrm{yr}^{-1}$ (Heinke et al. 2013; Van et al. 2019; the quoted value is scaled to canonical neutron star).
20. SAX J1808.4-3658. From different estimates of the upper limit on $\tilde{L}_{\mathrm{q}}$ given by Heinke et al. (2009) $\left(4.9 \times 10^{30} \mathrm{erg} \mathrm{s}^{-1}\right.$, $6.2 \times 10^{30} \mathrm{erg} \mathrm{s}^{-1}$, and $1.3_{-0.8}^{+0.6} \times 10^{31} \mathrm{erg} \mathrm{s}^{-1}$ ), corresponding to different spectral models applied to fit the continuum, we have selected the highest estimate as the most conservative option.

21. XTE J1807-294. More precisely, $\left\langle\dot{M}_{\text {obs }}\right\rangle<\left(1.3_{-1.0}^{+1.7}\right) \times$ $10^{-11} M_{\odot} \mathrm{yr}^{-1}$ (Van et al. 2019; here it is scaled to the canonical neutron star model).

24. IGR J00291+5934. The given value of accretion rate is calculated using Eq. (13) by summation of the fluences listed in Table 2 of De Falco et al. (2017) for the four last outbursts, divided by the time interval covering these outbursts and preceding periods of quiescence $(\Delta t=13.8 \mathrm{yr}$ from September 2001 to July 2015), for the distance $4.2 \mathrm{kpc}$ derived by these authors. This estimate agrees well with $\left\langle\dot{M}_{\text {obs }}\right\rangle \approx 2.5 \times 10^{-12} M_{\odot} \mathrm{yr}^{-1}$ in Table 2 of Heinke et al. (2009), based on three outbursts, and is approximately twice as large as the rate reported by Van et al. (2019; presumably due to the lower distance estimate, 2.6$3.6 \mathrm{kpc}$, used in that paper) and approximately two times smaller than the rate reported by Coriat et al. (2012; likely due to the small interval of time averaging; $\Delta t \sim 3 \mathrm{yr}$ ). A substantial part of the quiescent emission is nonthermal, perhaps due to a residual accretion disk (Torres et al. 2008; Baglio et al. 2017).

25. HETE J1900.1-2455. The quoted estimate of $\tilde{L}_{\mathrm{q}}$ is based on an analysis of several nondetections and a single detection of this source in quiescence, carried out by Degenaar et al. (2017), who have shown that the crust may not have fully relaxed by the time of this detection (the likely quiescent base luminosity values are accommodated by the quoted uncertainties).

26. XTE J1701-462. We estimate $\left\langle\dot{M}_{\text {obs }}\right\rangle$ by multiplying outburst $\dot{M}$ from Table 1 of Turlione et al. (2015) by the outburst duration (1.6 years) and dividing by the fiducial time-line of X-ray observations (30 years). Since Turlione et al. (2015) noted that this source may not have reached equilibrium, we take the smallest observed luminosity as an upper bound on $\tilde{L}_{\mathrm{q}}$.

27. IGR J17480-2446 (Ter $5 X-2) .\left\langle\dot{M}_{\text {obs }}\right\rangle$ is estimated from the outburst level $\langle\dot{M}\rangle=3 \times 10^{-9}$ (11\% of the Eddington limit) multplied by the rough estimate of the duty cycle: 2 months of outburst in 2010 over observation timescale of $30 \mathrm{yr}$. We note that the spin frequency of this neutron star is relatively small $(11 \mathrm{~Hz}$, Papitto et al. 2011), suggesting that the total accreted mass is probably small and the crust may not be fully replaced yet by accreted material (Wijnands et al. 2013).

28. EXO 0748-676. The quoted $\tilde{L}_{\mathrm{q}}$ corresponds to the last observation reported by Degenaar et al. (2014). It should be noted that the same reference reports pre-outburst detection in 1980 by Einstein observatory with $\tilde{L}=2.3 \pm 1.2 \mathrm{erg} \mathrm{s}^{-1}$, which is compatible with the quoted estimate at the $\approx 1.3 \sigma$ level.

29. 1RXS J180408.9-342058. Luminosity is estimated on the basis of the temperature confidence interval in Table 2 of Parikh et al. (2018) for observation 3 (2.4 yr after the end of outburst) and fit with unfixed power-law index, for the assumed neutron star mass $M=1.6 M_{\odot}$ and radius $R=11 \mathrm{~km}$. It agrees with the $\mathrm{X}$-ray luminosity value in this table with bolometric correction.

30. Swift J174805.3-244637 (Ter 5 X-3). The estimate of $\left\langle\dot{M}_{\text {obs }}\right\rangle$ (Bahramian et al. 2014) includes an outburst of 


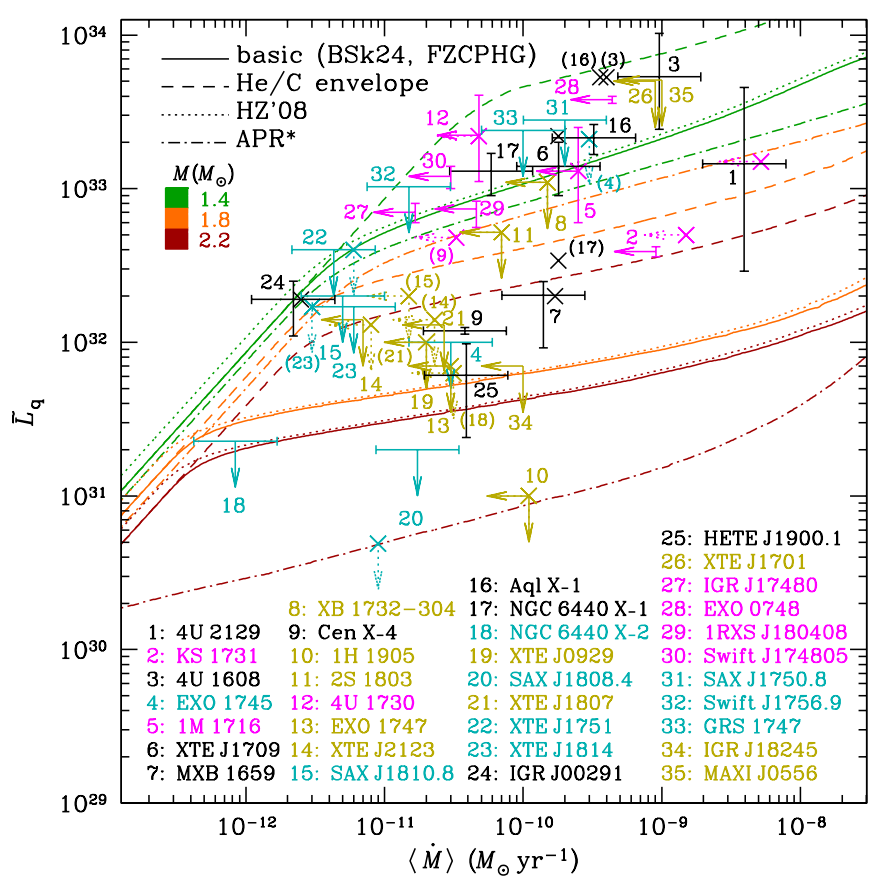

Fig. 3. Quiescent thermal luminosities of SXTs as functions of average accretion rates. Solid errorbars and arrows show the data listed in Table 2. Crosses without errorbars and dotted arrows show the older estimates and upper limits (e.g., Table 2 of Beznogov \& Yakovlev 2015a). The solid errorbars (arrows) are labeled by numbers from the first column of Tables 1 and 2, and abbreviated names of associated objects are listed in the lower part of the figure. In some cases, to avoid confusion, the crosses or dotted arrows are also labeled by the numbers in parentheses. The lines show theoretical predictions for the thermal quasi-equilibrium luminosity as a function of time-averaged accretion rate due to the heating of a fully accreted crust of neutron stars of three masses, $M=1.4 M_{\odot}, 1.8 M_{\odot}$, and $2.2 M_{\odot}$ (from upper to lower lines of the same type, coded by color), computed using the method described in Sect. 3.2 for different theoretical models described in Sect. 2: the "basic model" (solid lines), the same model with a fully accreted heatblanketing envelope (dashed lines), the basic model with HZ'08 heating and composition of the crust instead of FZCPHG (the dotted lines), and the alternative model (APR ${ }^{*}$ EoS and composition, BBST effective baryon masses) with iron thermal-insulating envelope (dot-dashed lines).

2002, which is not firmly attributed to this source; otherwise it could be smaller. For $\tilde{L}_{\mathrm{q}}$, we take the coldest measurement from Degenaar et al. (2015), which agrees with the pre-outburst level.

33. Swift J1750.7-3117 (GRS 1747-312). According to Vats et al. (2018), about half of the observed flux is thermal; perhaps there is a residual accretion.

34. IGR J18245-2452. This object is known to switch between accretion- and rotation-powered pulsar states (transitional millisecond pulsar, Papitto et al. 2013).

35. MAXI J0556-332. $\left\langle\dot{M}_{\text {obs }}\right\rangle$ is estimated as Eddingtonlimited accretion during 480 days of outburst (Homan et al. 2014) averaged over 30 years of X-ray observation time-line. For the luminosity, we take the minimal value from several observations of Parikh et al. (2017) and treat it as the upper bound, because the quasi-equilibrium state may not have yet been reached.

Figure 3 shows the redshifted thermal quasi-equilibrium luminosities of the neutron stars in the SXTs in quiescence, $\tilde{L}_{\mathrm{q}}$, and their average accretion rates, $\left\langle\dot{M}_{\text {obs }}\right\rangle$, inferred from observations. The estimates of $\tilde{L}_{\mathrm{q}}$ and $\left\langle\dot{M}_{\text {obs }}\right\rangle$ are plotted in Fig. 3 as error bars, and the upper bounds are indicated by arrows. The errors are provisionally set to a factor of two in the average accretion rates. Such error bars appear to approximately represent the anticipated magnitude of cumulative statistical and systematic errors. For the luminosities, we use errors from Table 2, which do not include possible systematic uncertainties (except when especially noted). The most important sources of error in most cases appear to be, for $\langle\dot{M}\rangle$, the lack of reliable observations on a long time-line and in some cases the uncertainty on the distance ${ }^{3}$, and for $L_{\mathrm{q}}$, the uncertainty on the distance, to which in some cases are added uncertainties in spectral decomposition and emission models. For comparison, along with the data from Table 2 we plot the traditional dataset, which has been used for similar illustrations up to now (Heinke et al. 2010; Wijnands et al. 2013, 2017; Beznogov \& Yakovlev 2015a,b; Fortin et al. 2018). In the cases where both $\langle\dot{M}\rangle$ and $L_{\mathrm{q}}$ have been estimated, the error bars are plotted in black, while different colors are chosen to show the cases where one of these quantities or both of them have only upper limits. In the bottom of the figure, truncated names of the SXT sources are listed for easy reference. The lines in Fig. 3 show theoretical functions $\tilde{L}(\langle\dot{M}\rangle)$ explained in Sect. 3.2.

\subsection{Simple evaluation of quiescent luminosity}

The timescale of thermal relaxation of neutron-star crust is much shorter than the neutron-star cooling timescale (see, e.g., Gnedin et al. 2001). Therefore, after the accretion halts, the neutron star relaxes to thermal quasi-equilibrium, which is determined by neglecting the slow variations of the thermal state of the stellar core (cf. Colpi et al. 2001). The quasi-equilibrium temperature distribution is controlled by the redshifted temperature of the core $\tilde{T}_{\text {core }}$, which is nearly constant because of the high thermal conductivity in the core. Therefore, a quiescent state of a neutron star in an SXT should be the same as the state of a cooling isolated neutron star (INS) with the same mass and composition at the age when this virtual INS would have the same $\tilde{T}_{\text {core }}$ as the considered neutron star in the SXT at quiescence.

This similarity is often used to determine quasi-equilibrium quiescent thermal luminosities of the SXTs, following the method suggested by Yakovlev et al. (2003). This method assumes that the total energy loss by a neutron star in the quasi-equilibrium state is equal to the heat deposited by the deep crustal heating over a period covering many cycles of outbursts and quiescence. This assumption can be written as $\tilde{L}_{\text {tot }}=\left\langle\tilde{L}_{\mathrm{h}}\right\rangle$, where

$\tilde{L}_{\mathrm{tot}}=\tilde{L}_{v}+\tilde{L}_{\gamma}$

which is the total energy loss in unit time as measured by a distant observer, $\tilde{L}_{\mathrm{h}}$ and $\tilde{L}_{v}$ are the redshifted heating power and neutrino luminosity given by Eq. (10), and $\tilde{L}_{\gamma}$ is the measured bolometric photon luminosity of the INS, which is assumed to be equal to the quasi-equilibrium measured bolometric photon luminosity of the SXT in quiescence, $\tilde{L}_{\mathrm{q}}$.

This method is illustrated in Fig. 4. Here, the average heating power is calculated according to the model FZCPHG, using Eq. (10), which in the case of heat sources concentrated at a

As recently shown by Carbone \& Wijnands (2019), a bias can be associated with estimation of the duty cycle (that is the fraction of time in the active state): missing of the outbursts decreases estimated duty cycle for systems with rare outbursts, while variability of the duty cycle can lead to overestimation of the duty cycle for systems with frequent outbursts. 
series of thin shells turns into

$\tilde{L}_{\mathrm{h}}=\sum_{i} \frac{\mathrm{e}^{2 \Phi_{i} / c^{2}}}{\sqrt{1-2 G M_{\mathrm{r}, i} / r_{i} c^{2}}} W_{i}$,

where $i$ enumerates the reaction shells in the order of increasing pressure, $r_{i}$ is the radius at the given shell, $M_{\mathrm{r}, i}, \Phi_{i}$, and $W_{i}$ are the respective values of $M_{\mathrm{r}}, \Phi(r)$, and heating power generated at the given surface. The last quantity is given by the relation

$$
\begin{aligned}
W_{i}=\dot{a} \cdot E_{\mathrm{h}, i} & =3.8 \times 10^{49}\left(\dot{M} / M_{\odot} \mathrm{yr}^{-1}\right) E_{\mathrm{h}, i} \mathrm{~s}^{-1} \\
& \approx 6 \times 10^{34} \dot{M}_{-9}\left(E_{\mathrm{h}, i} / \mathrm{MeV}\right) \mathrm{erg} \mathrm{s}^{-1},
\end{aligned}
$$

where $\dot{a}$ is the number of accreted baryons per unit time, $E_{\mathrm{h}, i}$ is the released energy per baryon at the $i$ th reaction shell, and $\dot{M}_{-9}$ is the accretion rate in units of $10^{-9} M_{\odot} \mathrm{yr}^{-1}$ in the local reference frame. The summation in Eq. (18) is performed only for those shells that lie within the accreted part of the crust, which means that the total mass above a given shell, $\Delta M=M-M_{\mathrm{r}, i}$, is smaller than the total accreted mass $\Delta M_{\text {acc }}$. Here and hereafter, following the previous works, we neglect the heat that is released due to compression of the pristine ground-state crust in the course of accretion, assuming it to be smaller than the heat produced by the nuclear reactions in the accreted crust.

The lines in Fig. 3 show theoretical redshifted thermal quasiequilibrium luminosities in quiescence, $\tilde{L}_{\mathrm{q}}$, as functions of the average accretion rate. The luminosities have been computed under the assumption of fully accreted crust, which provides the maximum deep heating power by including all the reaction shells in the sum in Eq. (18). The physics input is described in Sect. 2. Our basic model includes the BSk24 EoS and composition of the nonaccreted part of the star, FZCPHG model of heating and composition of the accreted crust, the MSH, BS, and BEEHS models for different types of superfluidity, and the iron heat-blanketing envelope. The alternative model, labeled APR*, employs the APR* EoS and composition of the core and the BBST effective masses of the nucleons. Additional modifications include allowance for the accreted heat-blanketing envelopes composed of helium and carbon instead of iron (see, e.g., Potekhin et al. 1997; Beznogov et al. 2016) or the use of the HZ'08 model for the composition and heating of the accreted crust. The accretion rates in the local reference frame, $\dot{M}$, have been used as input for obtaining the heating power density $Q_{\mathrm{h}}$, but in the figure they have been converted into $\dot{M}_{\text {obs }}$ according to Eq. (16) (with $A=1$ ) for direct comparison with the data listed in Table 2.

A comparison of the theoretical heating curves and the observational data in Fig. 3 shows that the quiescent luminosities of the hottest sources in the upper part of the figure can only be explained if we suppose that they have accreted heat blanketing envelopes, whereas one of the coldest SXTs, SAX J1808.4-3658, requires a very massive neutron star for its explanation and is not compatible with some theoretical models of the neutron star matter: in our case, it can be described by the APR* model of a neutron star with mass $M=2.2 M_{\odot}$ and iron envelope, but not by the BSk24 model. This difference is related to the larger stiffness of the BSk24 EoS, which leads to smaller central densities of the most massive neutron star models and consequently to lower intensities of the direct Urca process, compared to the $\mathrm{APR}^{*}$ models.

In Fig. 4, various neutron-star luminosities are plotted as functions of time $t$, assuming the simplified model, in which accretion proceeds at a constant average rate $\langle\dot{M}\rangle$ and starts sufficiently soon after the start of the cooling that the difference

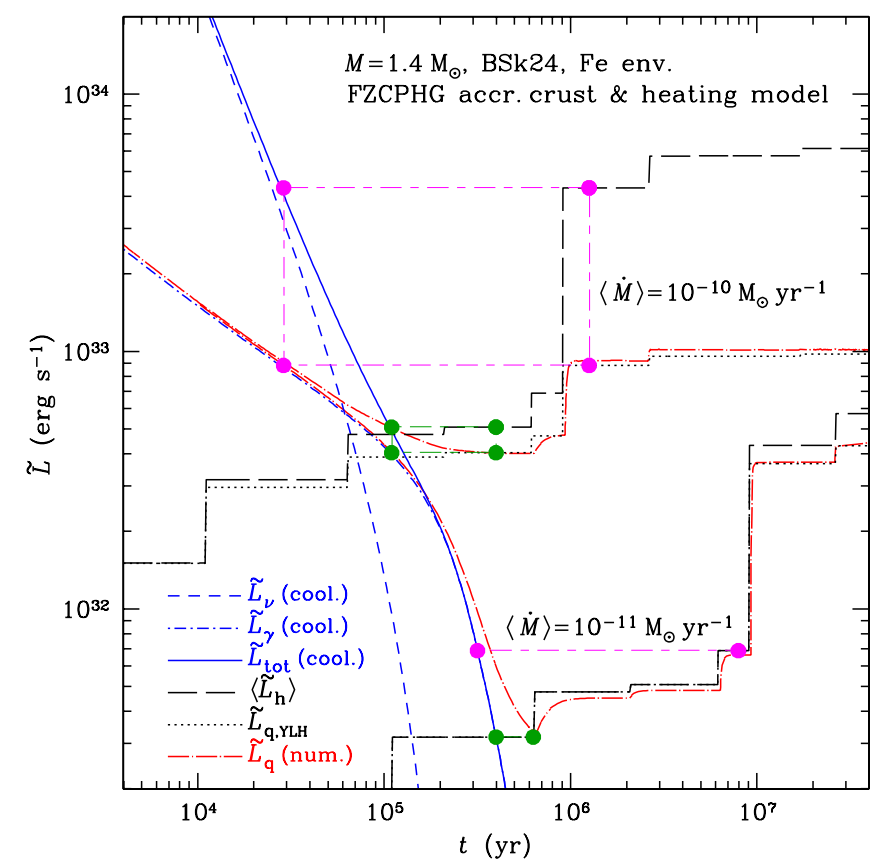

Fig. 4. Illustration of the approximate calculation of the quasiequilibrium quiescent luminosity according to the YLH method for a neutron star with mass $M=1.4 M_{\odot}$, described by the BSk24 EoS and composition model in the nonaccreted crust and the core and by the FZCPHG model of composition and heat sources in the accreted crust, with an iron heat-blanketing envelope. The blue solid line shows the total energy loss rate $\tilde{L}_{\text {tot }}$, which is the sum of the neutrino luminosity $L_{v}$ (blue short-dashed line) and the photon luminosity $L_{\gamma}$ (blue dotshort-dash line), of a cooling INS as function of the cooling time $t$. The black long-dashed stepped lines show the average power $\left\langle\tilde{L}_{\mathrm{h}}\right\rangle$ as function of the accretion time $t$, assuming a constant average accretion rate $\langle\dot{M}\rangle=10^{-10} M_{\odot} \mathrm{yr}^{-1}$ (the upper line) or $\langle\dot{M}\rangle=10^{-11} M_{\odot} \mathrm{yr}^{-1}$ (the lower line). The dotted stepped lines show the photon luminosities $\tilde{L}_{\gamma}$, as functions of the accretion time, which correspond to the cooling time moments when $\tilde{L}_{\text {tot }}=\left\langle\tilde{L}_{\mathrm{h}}\right\rangle$. The thin long-dash-short-dash lines serve as guides to the eye: they connect the corresponding total and photon luminosities and the corresponding cooling and heating time values. The red dot-long-dashed lines show the evolution of the bolometric photon luminosity in the numerical model of a cooling and heating neutron star, assuming that the accretion starts sufficiently soon after the start of the cooling and proceeds at a rate either $10^{-10} M_{\odot} \mathrm{yr}^{-1}$ (the upper curve) or $10^{-11} M_{\odot} \mathrm{yr}^{-1}$ (the lower line). All plotted luminosities are redshifted as measured in a remote frame of reference.

between the cooling age and accretion duration can be neglected. In this case $\Delta M_{\text {acc }}=\langle\dot{M}\rangle t$. Most of the time-dependences shown below imply these minimal assumptions. The lines corresponding to two fixed values of the average accretion rate are shown, $\langle\dot{M}\rangle=10^{-10} M_{\odot} \mathrm{yr}^{-1}$ and $\langle\dot{M}\rangle=10^{-11} M_{\odot} \mathrm{yr}^{-1}$.

For each accretion rate, one of the lines shows the average redshifted heating power $\tilde{L}_{\mathrm{h}}$. The "steps" on this line correspond to the moments $t$, when the accreted matter starts to involve a new reaction shell, meaning that a new discrete heat source is included in the sum (18); $\tilde{L}_{\mathrm{h}}$ is constant between these moments, so the line is horizontal.

In the same figure, we have plotted $\tilde{L}_{v}, \tilde{L}_{\gamma}$, and $\tilde{L}_{\text {tot }}$, that is, Eq. (17), for a cooling neutron star as functions of the cooling time and the photon luminosity in quiescence $\tilde{L}_{\mathrm{q}}$, calculated according to the Yakovlev et al. (2003; YLH) method. In this case, the quasi-equilibrium luminosity in quiescence $\tilde{L}_{\mathrm{q}}$ increases in steps as a function of the accretion time, following the steps of $\tilde{L}_{\mathrm{h}}$. The maximum $\tilde{L}_{\mathrm{q}}$ value is reached 


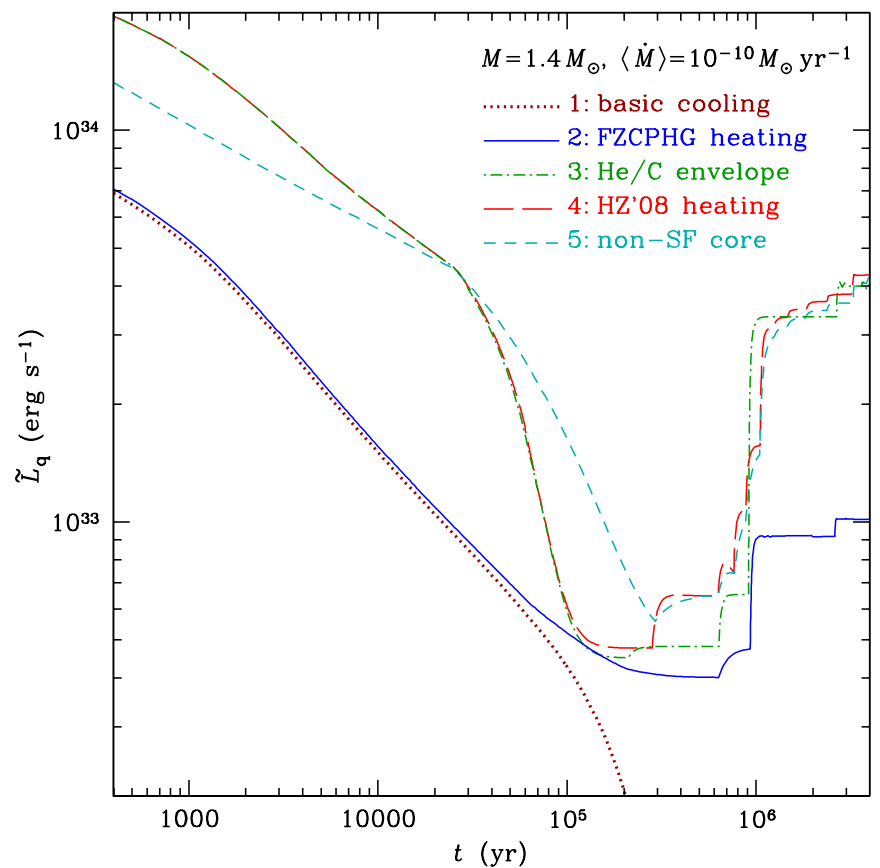

Fig. 5. Illustration of the effects of differences in the physics input on the long-term evolution of a neutron star. Redshifted thermal luminosity is shown as a function of time. Dotted line (1) - Basic cooling model. Solid line (2) - Same model with heating according to the FZCPHG model, calculated assuming that the accretion starts shortly after the birth of the neutron star with constant average rate of $10^{-10} M_{\odot} \mathrm{yr}^{-1}$. Dot-dashed line (3) - Same with replacement of the iron heat-insulating envelope to an accreted $\mathrm{He} / \mathrm{C}$ envelope. Long-dashed line (4) - Same accreted envelope, but the alternative heating model. Short-dashed line (5) - Same but without baryon superfluidity in the core.

when the innermost reaction shell has become included in the accreted crust. In the FZCPHG model this occurs when the accreted matter is pushed to the density of the second to last reaction shell $\rho=1.7 \times 10^{13} \mathrm{~g} \mathrm{~cm}^{-3}$. Pushing it further to the last shell at $\rho=7.3 \times 10^{13} \mathrm{~g} \mathrm{~cm}^{-3}$ does not increase $L_{\mathrm{h}}$, because the heating power $W$ at the last shell is negligible. For the neutron star model in Fig. $4\left(M=1.4 M_{\odot}\right.$, the BSk24 and FZCPHG models for the nonaccreted and accreted matter, respectively), the saturation of the heating power occurs at accretion time $t \approx 1.7 \times 10^{-3}\left(\langle\dot{M}\rangle / M_{\odot}\right)^{-1}$. At earlier epochs, the heating power and the respective quiescent luminosity are smaller.

\section{Long-term thermal evolution}

We have compared the YLH method with the results of our accurate numerical simulations of the evolution of a cooling and heating neutron star. Figure 4 presents the bolometric photon luminosity as a function of time. In the numerical model, the photon luminosity decreases at early age when it is dominated by the heat initially stored in the interior of the neutron star and the crust has a mainly ground-state composition, meaning that the deep crustal heating is negligible. The luminosity has a minimum at an intermediate age $\sim 10^{5}-10^{6} \mathrm{yr}$ (depending on $\langle\dot{M}\rangle$ ) and then increases due to the increasing thickness of the accreted part of the crust. We assume that the crust is initially groundstate, but that it is gradually being replaced by the accreted crust. The boundary between the accreted crust and the ground-state crust is determined by the accreted mass $\Delta M_{\text {acc }}=\langle\dot{M}\rangle t$. When the reprocessed accreted matter reaches a new reaction shell, $\tilde{L}_{\mathrm{q}}$

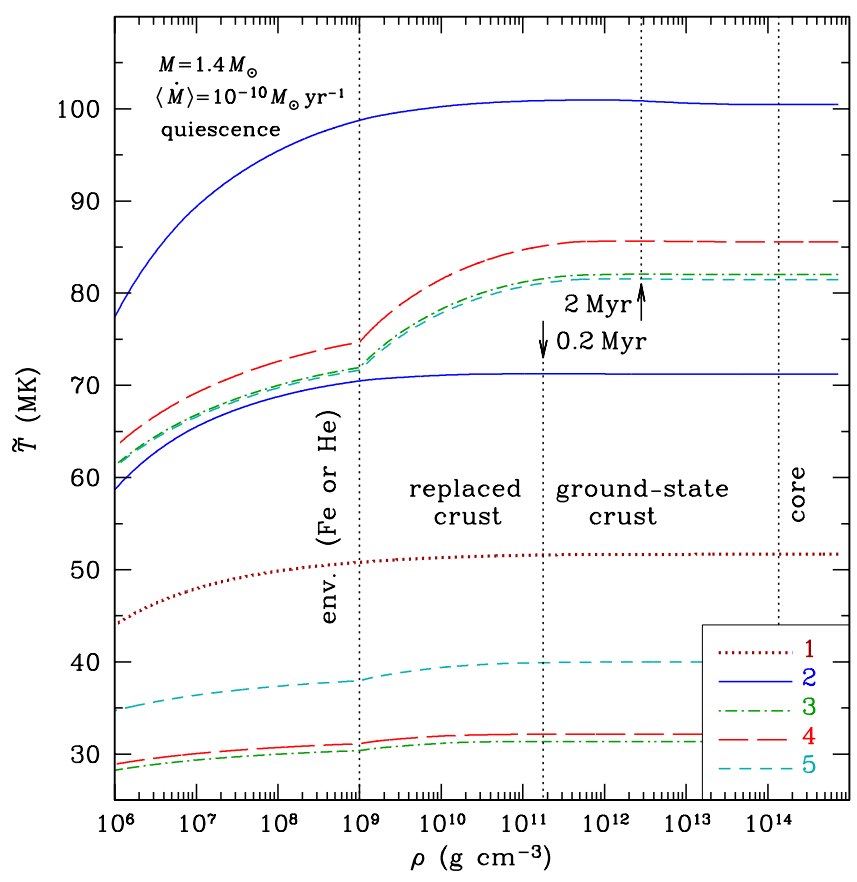

Fig. 6. Redshifted temperature profiles for the same models as in Fig. 5 drawn with the same line styles at $t=2 \times 10^{5} \mathrm{yr}$ (the lower curves) and $t=2 \times 10^{6} \mathrm{yr}$ (the upper curves). The vertical dotted lines separate the iron or accreted envelope, the accreted (replaced) crust, the nonaccreted (ground-state) crust, and the core.

starts to increase, first sharply and then slowly approaching the new quasi-stationary value. The comparison shows that the YLH model accurately predicts the quasi-stationary values of the redshifted bolometric luminosity in quiescence, $\widetilde{L}_{\mathrm{q}}$, although it does not reproduce details of transitions from one quasi-stationary value to the next. In reality, $\tilde{L}_{\mathrm{q}}$ does not immediately follow $\tilde{L}_{\mathrm{h}}$. Instead, it gradually approaches the equilibrium values predicted by the YLH model. With increasing accretion time, this delay becomes decreasingly significant (in comparison with the age of the star), so that for the old SXTs the YLH method proves to be very accurate. We also see that the minimum value $\tilde{L}_{\mathrm{q}, \min }$ is rather accurately determined by the intersection of the stepped line representing the YLH model and the INS cooling curve.

In Fig. 5 we examine the effects of several alterations in the models of outer envelopes, crust, and core, and two different heating models on the long-term average evolution of the thermal luminosity of an accreting neutron star of the "canonical" mass $M=1.4 M_{\odot}$. Here, the thermal evolution computed using the same model as in Fig. 4 is compared to the analogous computations but with replacement of some ingredients of the theoretical model to their alternatives.

First, we include an accreted envelope instead of the standard iron envelope, which extends to $\rho=10^{9} \mathrm{~g} \mathrm{~cm}^{-3}$. The lightelement blanketing envelope is more transparent to heat, and therefore the surface luminosity is higher. Subsequently, keeping the accreted envelope unchanged, we replace the FZCPHG accreted-crust model by the HZ'08 model. The effect of this replacement is noticeable, although less dramatic than the effect of the accreted outer envelope. We then explore the effects of the superfluidity and the effective baryon masses. A change of the superfluidity model in the crust from MSH to GIPSF and a change of the baryon effective mass model have almost no effect on the thermal evolution, meaning that the corresponding curves would be practically indistinguishable if plotted in Fig. 5. 


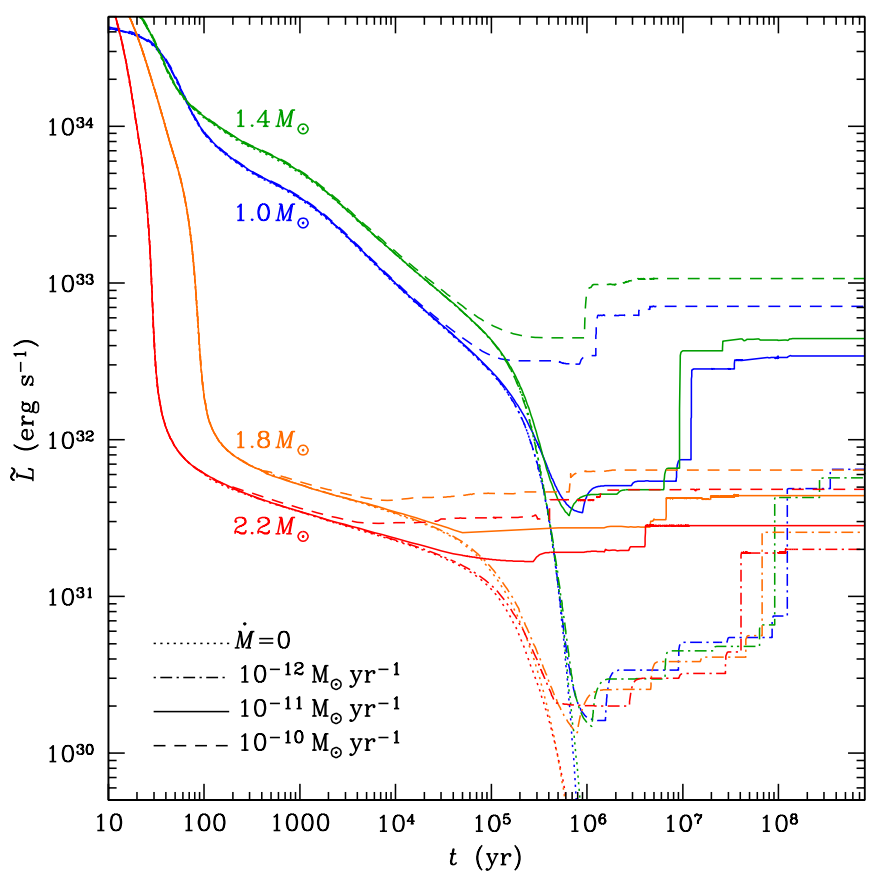

Fig. 7. Examples of the long-term evolution of the quasi-steady thermal luminosities for different neutron star masses (coded with colors and labeled near the curves) and different average mass accretion rates (shown with different line styles, according to the legend).

In contrast, switching off superfluidity in the core is seen to have a substantial effect, particularly at the cooling stage.

Figure 6 shows the corresponding internal temperature profiles at two moments of time, $200 \mathrm{kyr}$ and $2 \mathrm{Myr}$. One can see the breaks on the profiles 3-5 at density $\rho=10^{9} \mathrm{~g} \mathrm{~cm}^{-3}$, which limits the helium-accreted crust in these three models: the smaller slopes of the lines reflect the lower thermal conductivity for lighter chemical elements. In this figure we also mark the boundaries of the replaced accreted crust layer, where the heating sources are confined. The thickness of this layer is larger for the larger accretion duration (2Myr) than for the smaller one (200 kyr), therefore it includes more heating sources (cf. Fig. 1), which explains the higher positions of the temperature profiles for $t=2 \mathrm{Myr}$. The higher position of profile 2, calculated assuming the iron heat-blanketing envelope, is explained by the better thermal insulation provided by this envelope. The same insulation results in cooler surface layers (beyond the figure frame) and the lower photon flux seen in Fig. 5.

Figure 7 illustrates the influence of neutron star mass $M$ and average accretion rate $\langle\dot{M}\rangle$ on the long-term evolution of the thermal luminosity in quiescence. It is computed for the basic model with the accreted FZCPHG crust, which gradually replaces the ground-state BSk 24 crust. The direct Urca processes are forbidden for the two lower masses shown in the figure and open for the two higher masses. Accordingly, these massive stars cool down quickly via neutrino emission and have smaller thermal photon luminosities.

We see that, under the assumption of constant average accretion rate, the long-term evolution of the quiescent thermal luminosity is nonmonotonous. After initial cooling, it has a minimum and then increases due to continued accumulation of the accreted matter and activation of deeper reaction shells in the crust. In reality, the accretion rate can vary. For instance, accretion can start after a long period of pure cooling. In this case, the minimum of the luminosity can be much lower than shown in our figures.

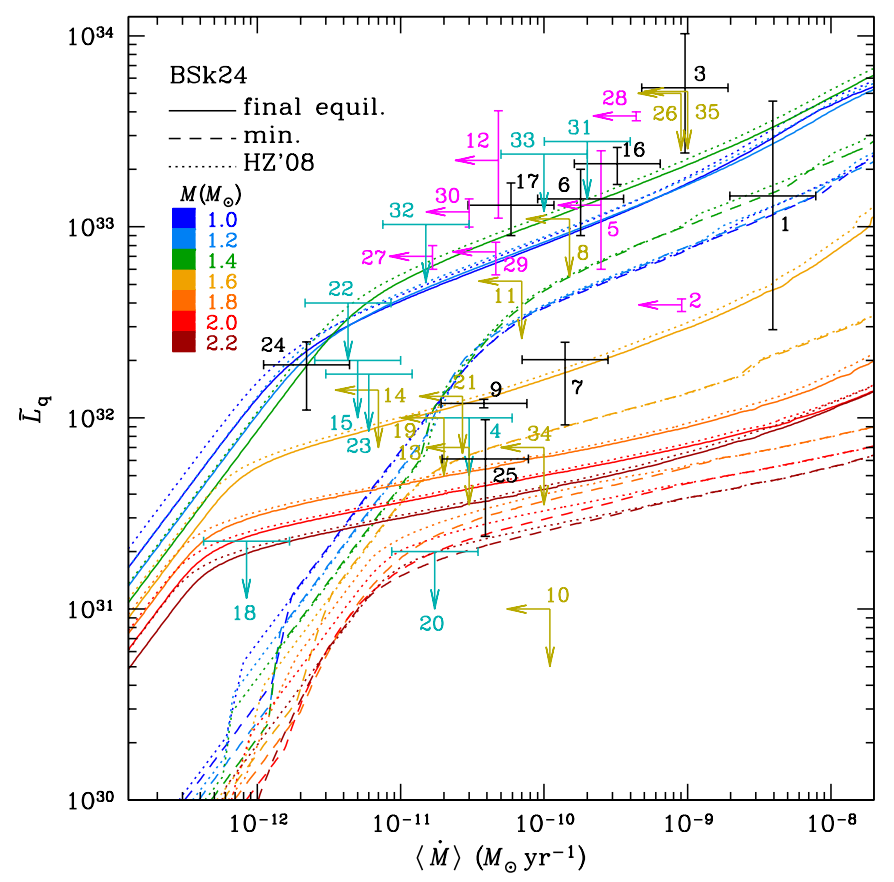

Fig. 8. Quasi-equilibrium redshifted luminosity as a function of the average mass accretion rate $\langle\dot{M}\rangle$ for different neutron star masses (coded with color) in the basic neutron-star model. Solid lines show the maximum luminosity $\tilde{L}_{\mathrm{q}}$ for the FZCPHG model of deep crustal heating, dashed lines show the minimum $\tilde{L}_{\mathrm{q}}$ for the same model, and dotted lines display the results for the HZ'08 accreted crust heating model (both maximum and minimum).

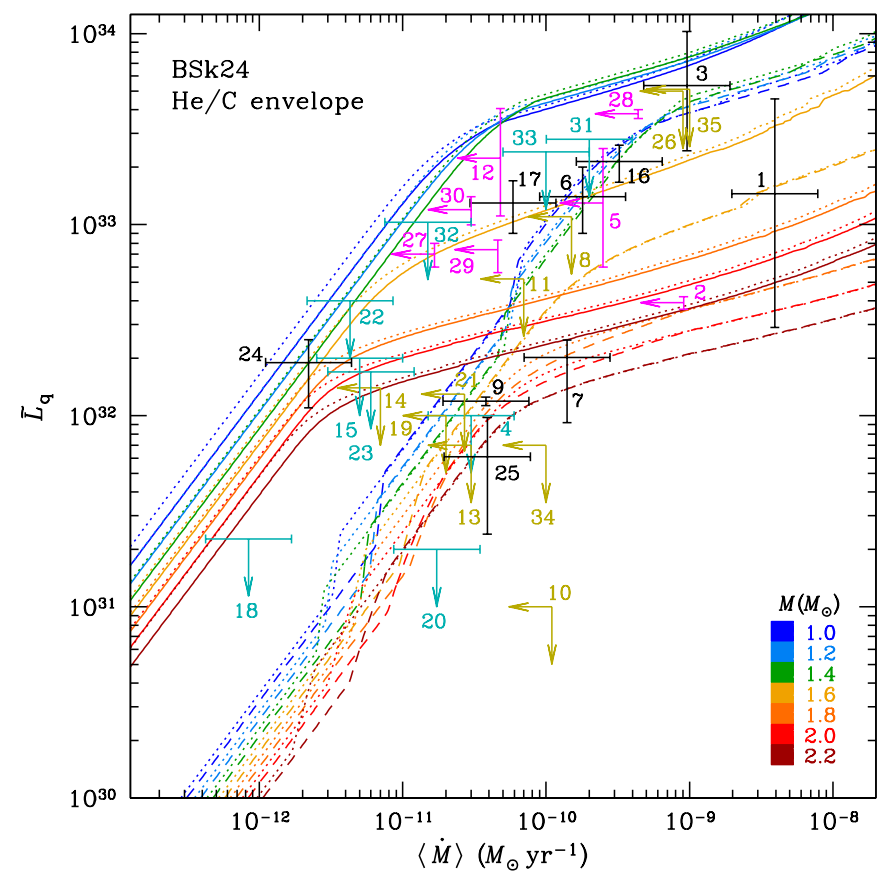

Fig. 9. Same as in Fig. 8 but for a light-element composition of the outer envelope from the surface to $\rho=10^{9} \mathrm{~g} \mathrm{~cm}^{-3}$.

In any case, the quiescent luminosity for a given SXT can have any value in a "window" between the minimum and the final quasi-steady state at any given average accretion rate. Coming back to the simplest assumption of constant $\langle\dot{M}\rangle$, we can calculate this window, which is sliding as a function of $\langle\dot{M}\rangle$. The results of such calculations are shown in Figs. 8-11 for the 


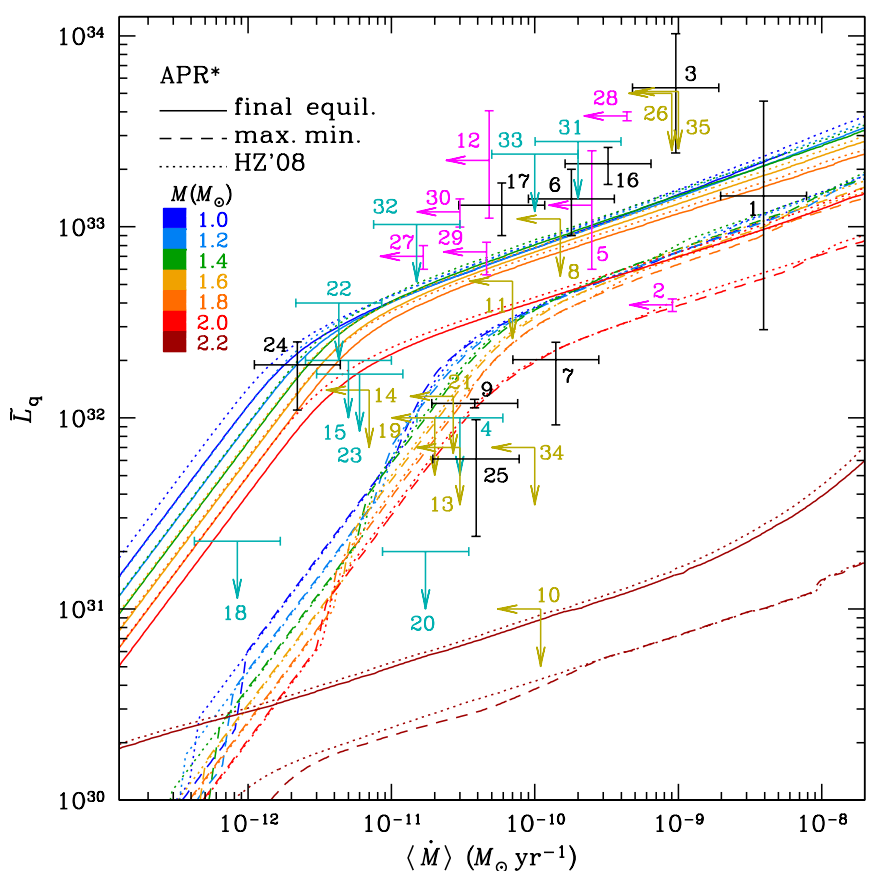

Fig. 10. Same as in Fig. 8 but for the APR* EoS, composition, and effective masses.

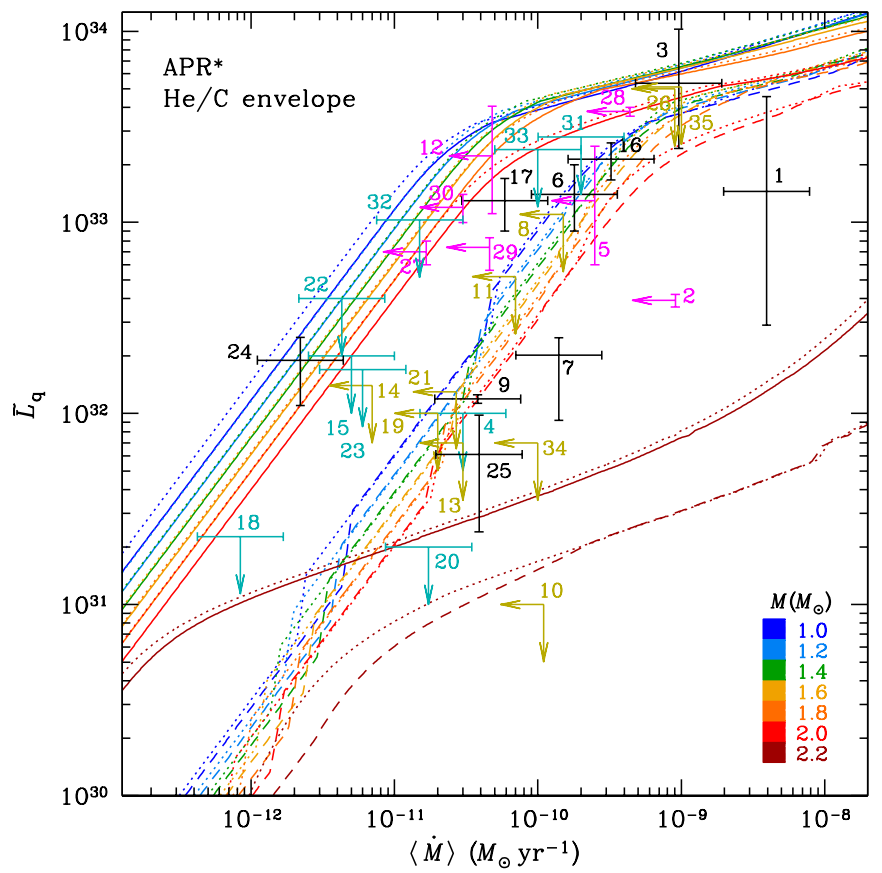

Fig. 11. Same as in Fig. 10 but with an accreted envelope as in Fig. 9.

BSk24 and APR* models of the interior with the traditional iron heat blanketing envelope and with heat-transparent $\mathrm{He} / \mathrm{C}$ envelope. We see that the basic model (Fig. 8) is unable to explain the whole range of the estimated values of $\tilde{L}_{\mathrm{q}}$ and $\langle\dot{M}\rangle$ simultaneously. At a given $\langle\dot{M}\rangle$, the hottest objects (numbers 3, 6, 12, 28 ) are brought to agreement with the theory by the assumption that their heat-blanketing envelopes are composed of light elements (Figs. 9 and 11). In addition, the masses of these hot objects should not exceed $M_{\mathrm{DU}}$. On the contrary, several of the coldest objects (numbers 4, 7, 20, 25) are better explained without the accreted envelopes and with $M>M_{\mathrm{DU}}$.
Object 20 (SAX J1808.4-3658) appears to be incompatible with the BSk24 model if its crust is entirely replaced by the accreted material. An upper bound on the quiescent luminosity of this SXT, evaluated from observations, restricts $\tilde{L}_{\mathrm{q}}$ to values that are very low for its estimated accretion rate. In the APR* model, it can be explained (Figs. 10 and 11), but only if its mass appreciably exceeds $2 M_{\odot}$. However, an analysis based on evolutionary scenarios (Tailo et al. 2018) favors $M \sim 1.6 M_{\odot}$, which agrees with results of Morsink \& Leahy (2011). If the crust is only partially replaced, so that the luminosity is near the minimum, then this SXT is compatible with the BSk24 model for any mass, but only marginally (Figs. 8 and 9). However, the mass of the donor star, estimated from observations, is very low, $M_{\mathrm{d}} \sim$ 0.04-0.07, (Wang et al. 2013; Sanna et al. 2016), which implies a large accreted mass $\Delta M_{\text {acc }} \sim 0.2 M_{\odot}$ (Tailo et al. 2018). The short spin period of this pulsar $(2.5 \mathrm{~ms})$ corroborates a large accreted mass and hence the fully replaced crust. In the following section we see that this difficulty is resolved, when one takes recent advances in the theory of baryon superfluidity into account.

\section{Effect of triplet baryon pairing suppression}

The superfluidity is known to affect neutron star thermal evolution (see Sect. 2.5). In particular, the powerful direct Urca processes, being open at $\bar{n}>\bar{n}_{\mathrm{DU}}$, can still be strongly suppressed by baryon superfluidity (Yakovlev et al. 2001). It is likely that in the core of a neutron star the proton singlet superfluidity is the strongest one (has the highest critical temperature), but only up to a density of $\rho \sim(3-5) \times 10^{14} \mathrm{~g} \mathrm{~cm}^{-3}$ (Fig. 2). At higher densities, the neutron-triplet superfluidity comes into play. These higher densities are most important for the neutrino emission by the direct Urca process. As a rule, the neutron-triplet superfluidity has a lower critical temperature than the proton singlet one. We note that even if the maximum triplet pairing gap is similar to that of the singlet type, $T_{\text {crit }}$ still is lower by a factor of approximately one fifth due to the anisotropy of the triplet gap (e.g., Amundsen \& Østgaard 1985; Baldo et al. 1992; cf. Ho et al. 2015; this factor of difference is sometimes overlooked in the literature on neutron star cooling). However, according to several recent studies (see Sedrakian \& Clark 2018, for review and references), many-particle correlations in the baryon matter lead to strong suppression of the triplet type of superfluidity. In particular, the results of Ding et al. (2016) suggest that the pairing gap may be reduced by an order of magnitude or even stronger at high densities, as illustrated in the middle panel of Fig. 2. To test the effect of this suppression, we have compared the neutron star cooling and heating for the neutron-triplet-type superfluidity models "Av18 SRC+P" and "N3LO SRC+P" by Ding et al. (2016) with the BEEHS model by Baldo et al. (1998).

The results of such simulations for a neutron star with $M=$ 1.4 $M_{\odot}$ are shown in Fig. 12. We see that the suppression of the neutron-triplet superfluidity delays cooling at the late time of evolution and increases thermal luminosity at sufficiently high average accretion rates. The PBF neutrino emission is most powerful at $T \sim T_{\text {crit }}$ and is effectively quenched by the decrease of $T_{\text {crit. }}$

Analogous comparison for a more massive neutron star is presented in Fig. 13. The principal difference of this case from the previous one is that the direct Urca process operates in such a star. We see that the suppression of the neutron-triplet superfluidity for the massive star has an opposite effect compared to the previous figure: the cooling is accelerated, and the heating phase shows lower luminosities. This is because the direct Urca 


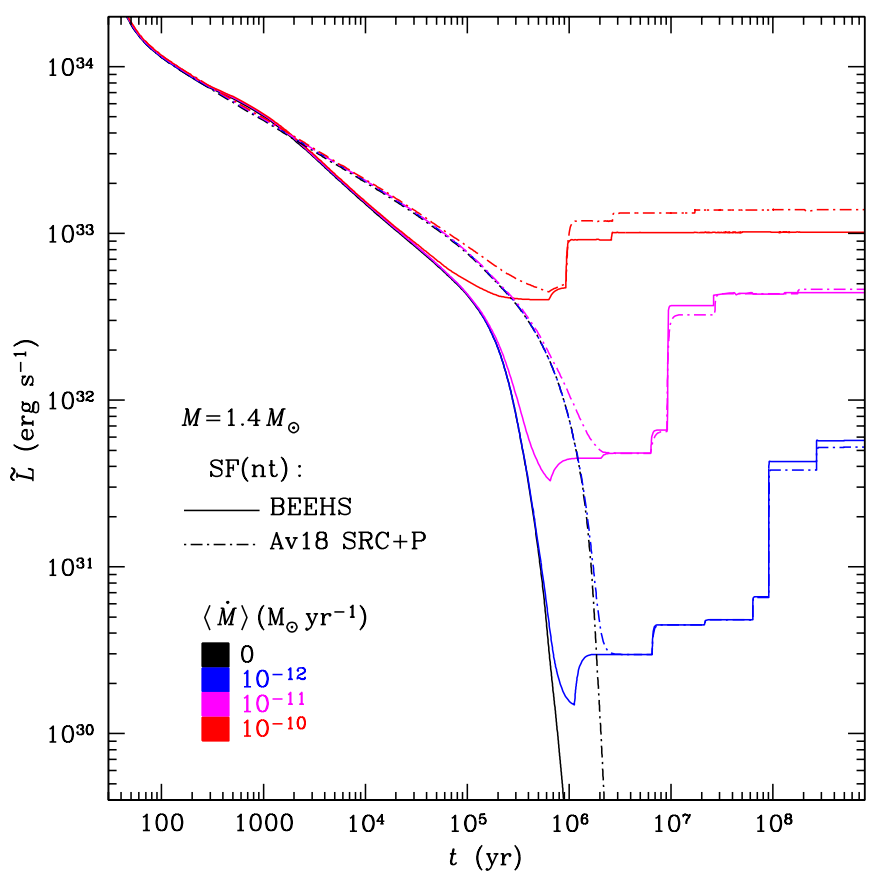

Fig. 12. Long-term evolution of the quasi-steady thermal luminosities for the basic model of a neutron star $\left(M=1.4 M_{\odot}\right.$, standard superfluidity) without accretion and with long-term steady accretion at different rates $\langle\dot{M}\rangle$ (solid lines), compared with evolution of the same star but with suppressed neutron-triplet superfluidity in the core (dot-dashed curves) according to the "Av18 SRC+P" pairing gap model of Ding et al. (2016).

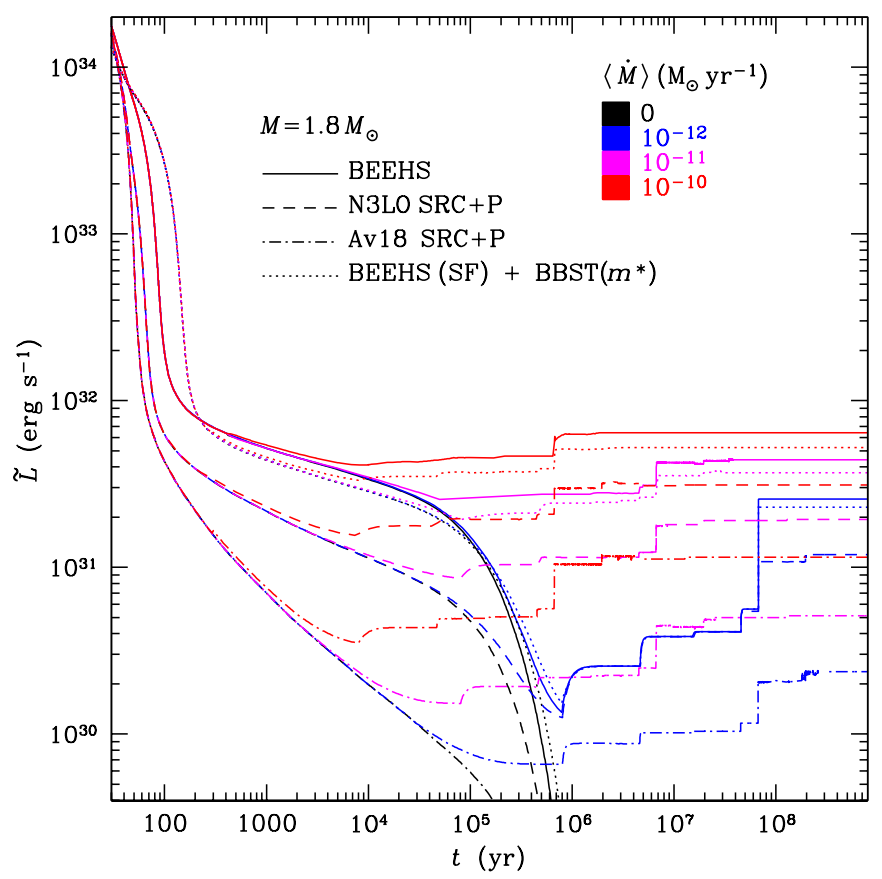

Fig. 13. Long-term evolution of the quasi-steady thermal luminosities for a neutron star with $M=1.8 M_{\odot}$ without accretion and with longterm steady accretion at different rates $\langle\dot{M}\rangle$ for different physics inputs in the core. The model with the standard nucleon superfluidity and effective masses consistent with the BSk24 EoS (solid lines) is compared with the results of using suppressed neutron-triplet superfluidity in the core according to models of Ding et al. (2016) "N3LO SRC+P" (dashed lines) and "Av18 SRC+P" (dot-dashed lines), or with the alternative model (BBST instead of BSk24) for the nucleon effective masses (dotted curves).

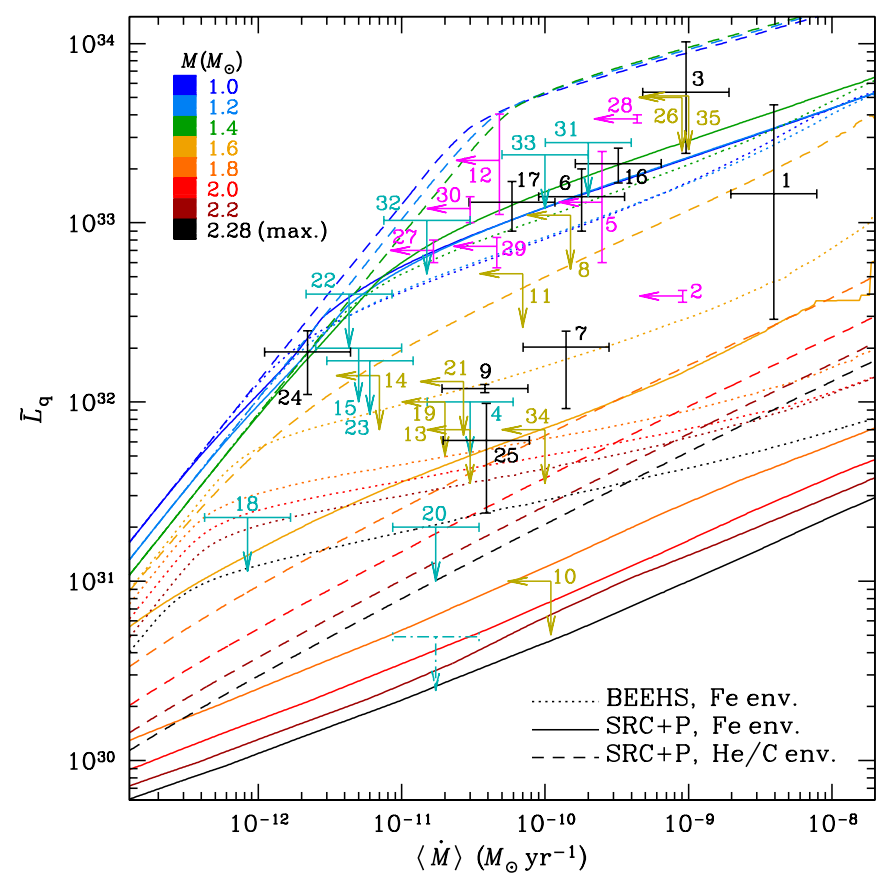

Fig. 14. Quasi-equilibrium redshifted luminosity as a function of the average observed mass accretion rate $\left\langle\dot{M}_{\text {obs }}\right\rangle$, computed including the effect of suppression of the neutron-triplet superfluidity. Solid lines are obtained for the iron heat-blanketing envelope and dashed lines for the accreted $(\mathrm{He} / \mathrm{C})$ envelope. For comparison, the dotted lines show the luminosities with nonsuppressed triplet superfluidity, as in Fig. 8. The solid error bars and arrows show the estimated values and upper limits listed in Table 2, as in the previous figures, and the additional dotdashed ones show the traditional tightest estimate of an upper bound on the thermal luminosity of SAX J1808.4-3658 in quiescence, but with the updated accretion rate.

emission fades away when baryon superfluidity develops. When superfluidity is partially suppressed, the direct Urca process partially regains its power. For comparison, in Fig. 13 we also show the thermal evolution computed with an alternative model of nucleon effective masses (BBST instead of BSk24, see Fig. 2). Since the effective masses affect the neutrino reaction rates, we see some differences at the stages of thermal evolution where the neutron star interior is sufficiently hot, meaning that the neutrino energy losses dominate over conductive losses. However, the dependence on the effective masses is seen to have a much smaller effect than the dependence on the superfluidity.

The heating curves for neutron stars of different masses with the partly suppressed neutron-triplet superfluidity are shown in Fig. 14 for the cases with iron and light-element heat blanketing envelopes. For comparison we also show the heating curves obtained with the nonsuppressed neutron-triplet superfluidity. We see that the suppression of the neutron-triplet superfluidity decreases the smaller thermal luminosities, appropriate to most massive neutron stars, and increases the higher luminosities, appropriate to neutron stars with lower masses. The first effect is explained by the fact that the direct Urca processes are strongly suppressed at $T \ll T_{\text {crit }}$. When $T_{\text {crit }}$ decreases, these processes take away more energy from the core and thus cool down the neutron star more efficiently. The second effect is due to the PBF mechanism. This mechanism of neutrino emission is entirely due to superfluidity, meaning that the partial suppression of superfluidity delays the PBF processes and thus preserves more heat inside the star. The lower heating curves are therefore pushed 
downward and the higher ones upward, which facilitates theoretical interpretation of the low and high values of $\tilde{L}_{\mathrm{q}}$. In particular, in this way we can explain not only the conservative upper limit on the quiescent thermal luminosity of one of the coldest transiently accreting neutron stars in SAX J1808.4-3658, but also the tightest nonconservative limit traditionally used in the literature (e.g., Beznogov \& Yakovlev 2015a), displayed against the updated estimate of the average accretion rate (Coriat et al. 2012; Van et al. 2019).

The hottest neutron stars in the SXTs still remain only marginally compatible with theoretical heating curves, computed in the models with iron heat-blanketing envelopes. However, the inclusion of an accreted outer envelope into the model increases the observed luminosities and thereby provides a simple explanation of all simultaneous estimates of $\tilde{L}_{\mathrm{q}}$ and $\left\langle\dot{M}_{\mathrm{obs}}\right\rangle$ in Table 2.

\section{Conclusions}

We have revisited the evaluation of quasi-equilibrium thermal luminosities of neutron stars in SXTs in quiescence, taking the recent progress in observations of the SXTs and in the theory of neutron stars into account. We have composed an updated collection of the key properties of SXTs with estimated average mass-accretion rates and neutron-star luminosities in quiescence. We have simulated long-term thermal evolution and computed thermal states of the SXTs with different mass accretion rates for different modern theoretical neutron-star models with the nucleon-lepton $($ nре $\mu)$ composition of the core. We explored the possibility that the crust of a neutron star is not completely replaced by the reprocessed accreted matter. In particular, we have computed the minimal quiescent thermal luminosities in the simplest model of an accretion at constant average rate. In this model, the minimal theoretical luminosity of the transiently accreting neutron stars that are relatively cool for their estimated average accretion rates becomes compatible with theory (albeit marginally) even without invoking the enhanced (direct Urca) cooling. However, their short spin periods (1.8-2.7 ms) suggest a large accreted mass and therefore disfavor such a scenario. Indeed, the evolutionary lifetime of a LMXB (gigayears) is longer than the time needed to accumulate the accreted mass $\Delta M_{\text {acc }} \gtrsim 0.002 M_{\odot}$ sufficient to reach the steady quiescent equilibrium, and is orders of magnitude longer than a megayear when the quiescent luminosity is at minimum. Therefore, the number of LMXB systems with neutron stars in this minimum state should be relatively small.

On the other hand, there are several transiently accreting neutron stars that are relatively hot for their estimated accretion rates, which can be explained by the presence of a relatively heat-transparent accreted outer envelope. Thus the updated observational data and updated theoretical physics input leave unchanged the basic conclusions of Yakovlev et al. (2003, 2004) on the possible explanations of the hottest and coldest neutron stars in SXTs. The replacement of the accreted crust model HZ'08 (Haensel \& Zdunik 2008) by the new model FZCPHG (Fantina et al. 2018) does not change any qualitative conclusions.

One of the coldest neutron stars in SXTs, SAX J1808.43658 , still presents a difficulty for theoretical interpretation. We have found that the difficulty is related to the suppression of the direct Urca process by neutron-triplet superfluidity in the core. Allowance for quenching of this type of superfluidity according to the results of Ding et al. (2016) makes the theoretical heating curves of this and other relatively cold transiently accreting neutron stars fully compatible with observations. Moreover, the same quenching brings the theory to better agreement with observed thermal luminosities of relatively hot transiently accreting neutron stars in quiescence, although the presence of an accreted envelope is still needed for such agreement. Thus the observational data on neutron-star heating in SXTs favor the suppression of the neutron triplet type of superfluidity, which is in line with the analogous conclusions made recently for the isolated cooling neutron stars (Beznogov et al. 2018; Wei et al. 2019).

Acknowledgements. We are grateful to A. D. Kaminker and P. S. Shternin for fruitful discussions and to P. Haensel and J. L. Zdunik for useful communications and for providing some details of their models of the accreted neutron-star crusts. The work of A.C. and A.P. was supported by the Russian Science Foundation (grant 19-12-00133).

\section{References}

Akmal, A., Pandharipande, V. R., \& Ravenhall, D. G. 1998, Phys. Rev. C, 58, 1804

Allen, J. L., Homan, J., Chakrabarty, D., \& Nowak, M. 2018, ApJ, 854, 58

Amundsen, L., \& Østgaard, E. 1985, Nucl. Phys. A, 444, 163

Baglio, M. C., Campana, S., D’Avanzo, P., et al. 2017, A\&A, 600, A109

Bahramian, A., Heinke, C. O., Sivakoff, G. R., et al. 2014, ApJ, 780, 127

Bahramian, A., Heinke, C. O., Degenaar, N., et al. 2015, MNRAS, 452, 3475

Baldo, M., \& Schulze, H.-J. 2007, Phys. Rev. C, 75, 025802

Baldo, M., Cugnon, J., Lejeune, A., \& Lombardo, U. 1992, Nucl. Phys. A, 536, 349

Baldo, M., Elgarøy, Ø., Engvik, L., Hjorth-Jensen, M., \& Schulze, H.-J. 1998, Phys. Rev. C, 58, 1921

Baldo, M., Burgio, G. F., Schulze, H.-J., \& Taranto, G. 2014, Phys. Rev. C, 89, 048801

Becker, W., Swarz, D. A., Pavlov, G. G., et al. 2003, ApJ, 594, 798

Beznogov, M. V., \& Yakovlev, D. G. 2015a, MNRAS, 447, 1598

Beznogov, M. V., \& Yakovlev, D. G. 2015b, MNRAS, 452, 540

Beznogov, M. V., Potekhin, A. Y., \& Yakovlev, D. G. 2016, MNRAS, 459, 1569

Beznogov, M. V., Rrapaj, E., Page, D., \& Reddy, S. 2018, Phys. Rev. C, 98, 035802

Bilous, A. V., Watts, A. L., Galloway, D. K., \& in 't Zand, J. J. M. 2018, ApJ, 862, L4

Bisnovatyi-Kogan, G. S. 2001, Stellar Physics 1. Fundamental Concepts and Stellar Equilibrium (Berlin: Springer), sect.1.4.6

Brown, E. F., Bildsten, L., \& Rutledge, R. E. 1998, ApJ, 504, L95

Brown, E. F., Cumming, A., Fattoyev, F. J., et al. 2018, Phys. Rev. L, 120, 182701 Bult, P., Patruno, A., \& van der Klis, M. 2015, ApJ, 814, 138

Cackett, E. M., Wijnands, R., Heinke, C. O., et al. 2005, ApJ, 620, 922

Cackett, E. M., Wijnands, R., Miller, J. M., Brown, E. F., \& Degenaar, N. 2008, ApJ, 687, L87

Cackett, E. M., Brown, E. F., Miller, J. M., \& Wijnands, R. 2010, ApJ, 720, 1325 Cackett, E. M., Brown, E. F., Cumming, A., et al. 2013, ApJ, 774, 131 Carbone, D., \& Wijnands, R. 2019, MNRAS, 488, 2767

Casares, J., Dubus, G., Shahbaz, T., Zurita, C., \& Charles, P. A. 2002, MNRAS, 329,29

Chakrabarty, D., \& Morgan, E. H. 1998, Nature, 394, 346

Chamel, N., Goriely, S., \& Pearson, J. M. 2009, Phys. Rev. C, 80, 065804

Chugunov, A. I., Gusakov, M. E., \& Kantor, E. M. 2014, MNRAS, 445, 385

Colpi, M., Geppert, U., Page, D., \& Possenti, A. 2001, ApJ, 529, L29

Coriat, M., Fender, R. P., \& Dubus, G. 2012, MNRAS, 424, 1991

Cornelisse, R., Wijnands, R., \& Homan, J. 2007, MNRAS, 380, 1637

Cornelisse, R., D’Avanzo, P., Campana, S., et al. 2012, MNRAS, 420, 3538

Cowley, A. P., Hutchings, J. B., Schmidtke, P. C., et al. 1988, AJ, 95, 1231

D’Avanzo, P., Campana, S., Israel, G. L., et al. 2005, A\&A, 444, 905

De Falco, V., Kuiper, L., Bozzo, E., et al. 2017, A\&A, 599, A88

Degenaar, N., \& Wijnands, R. 2012, MNRAS, 422, 581

Degenaar, N., Wijnands, R., \& Miller, J. M. 2013, ApJ, 767, L31

Degenaar, N., Medin, Z., Cumming, A., et al. 2014, ApJ, 791, 47

Degenaar, N., Wijnands, R., Bahramian, A., et al. 2015, MNRAS, 451, 2071

Degenaar, N., Ootes, L. S., Reynolds, M. T., Wijnands, R., \& Page, D. 2017,

MNRAS, 465, L10

Ding, D., Rios, A., Dussan, H., et al. 2016, Phys. Rev. C, 94, 025802

Douchin, F., \& Haensel, P. 2001, A\&A, 380, 151

Fantina, A. F., Zdunik, J. L., Chamel, N., et al. 2018, A\&A, 620, A105

Fiocchi, M., Natalucci, L., Chenevez, J., et al. 2009, ApJ, 693, 333

Fortin, M., Taranto, G., Burgio, G. F., Haensel, P., Schulze, H.-J., \& Zdunik,

J. L. 2018, MNRAS, 475, 5010

Friedman, J. L., \& Schutz, B. F. 1978a, ApJ, 221, 937 
Friedman, J. L., \& Schutz, B. F. 1978b, ApJ, 222, 281

Fridriksson, J. K., Homan, J., Wijnands, R., et al. 2011, ApJ, 736, 162

Fujimoto, M. Y., Hanawa, T., Iben, Jr., I., \& Richardson, M. B. 1984, ApJ, 315, 198

Galloway, D. K., Chakrabarty, D., Morgan, E. H., \& Remillard, R. A. 2002, ApJ, 576, L137

Galloway, D. K., Markwardt, C. B., Morgan, E. H., Chakrabarty, D., \& Strohmayer, T. E. 2005, ApJ, 622, L45

Galloway, D .K., Muno, M. P., Hartman, J. M., Psaltis, D. S., \& Chakrabarty, D 2008, ApJS, 179, 360

Gandolfi, S., Illarionov, A. Y., Pederiva, F., Schmidt, K. E., \& Fantoni, S. 2009, Phys. Rev. C, 80, 045802

Gnedin, O. Y., Yakovlev, D. G., \& Potekhin, A. Y. 2001, MNRAS, 324, 725

Goriely, S., Chamel, N., \& Pearson, J. M. 2010, Phys. Rev. C, 82, 035804

Goriely, S., Chamel, N., \& Pearson, J. M. 2013, Phys. Rev. C, 88, 024308

Haensel, P. 1995, Space Sci. Rev., 74, 427

Haensel, P., \& Potekhin, A. Y. 2004, A\&A, 428, 191

Haensel, P., \& Zdunik, J. L. 1990, A\&A, 229, 117

Haensel, P., \& Zdunik, J. L. 2003, A\&A, 404, 33

Haensel, P., \& Zdunik, J. L. 2008, A\&A, 480, 459

Haskell, B., Degenaar, N., \& Ho, W. C. G. 2012, MNRAS, 424, 93

Heinke, C. O., Jonker, P. G., Wijnands, R., \& Taam, R. E. 2007, ApJ, 660, 1424

Heinke, C. O., Jonker, P. G., Wijnands, R., Deloye, C. J., \& Taam, R. E. 2009, ApJ, 691, 1035

Heinke, C. O., Altamirano, D., Cohn, H. N., et al. 2010, ApJ, 714, 1894

Heinke, C. O., Ivanova, N., Engel, M. C., et al. 2013, ApJ, 768, 184

Ho, W. C. G., Elshamouty, K. G., Heinke, C. O., \& Potekhin, A. Y. 2015, Phys Rev. C, 91, 015806

Homan, J., Fridriksson, J. K., Wijnands, R., et al. 2014, ApJ, 795, 131

in 't Zand, J. J. M., Hulleman, F., Markwardt, C. B., et al. 2003, A\&A, 406, 233

Inogamov, N. A., \& Sunyaev, R. A. 2010, Astron. Lett., 36, 848

Jonker, P. G., \& Nelemans, G. 2004, MNRAS, 354, 355

Jonker, P. G., Galloway, D. K., McClintock, J. E., et al. 2004, MNRAS, 354, 666

Jonker, P. G., Bassa, C. G., \& Wachter, S. 2007a, MNRAS, 377, 1295

Jonker, P. G., Steeghs, D., Chakrabarty, D., \& Juett, A. M. 2007b, ApJ, 665, L147

Kaaret, P., Morgan, E. H., Vanderspek, R., \& Tomsick, J. A. 2006, ApJ, 638, 963

Krimm, H. A., Markwardt, C. B., Deloye, C. J., et al. 2007, ApJ, 668, L147, Erratum: 2009, ApJ, 703, L183

Kuulkers, E., den Hartog, P. R., in 't Zand, J. J. M., et al. 2003, A\&A, 399, 663

Lau, R., Beard, M., Gupta, S. S., et al. 2018, ApJ, 859, 62

Lasota, J.-P. 2001, New Astron. Rev., 449, 45

Leinson, L. B. 2010, Phys. Rev. C, 81, 025501

Li, Z. H., \& Schulze, H.-J. 2008, Phys. Rev. C, 78, 028801

Lin, D., Altamirano, D., Homan, J., et al. 2009, ApJ, 699, 60

Linares, M., Bahramian, A., Heinke, C., et al. 2014, MNRAS, 438, 251

Lowell, A. W., Tomsick, J. A., Heinke, C. O., et al. 2012, ApJ, 749, 11

Mackie, F. D., \& Baym, G. 1977, Nucl. Phys. A, 285, 332

Margueron, J., Sagawa, H., \& Hagino, K. 2008, Phys. Rev. C, 77, 054309

Markwardt, C. B., Swank, J. H., Strohmayer, T. E., in 't Zand, J. J. M., \& Marshall, F. E. 2002, ApJ, 575, L21

Matrange, M., Papitto, A., Di Salvo, T., et al. 2017, A\&A, 603, A39

Matsuo, Y., Liu, H., Hashimoto, M.-A., \& Noda, T. 2018, Int. J. Mod. Phys. E, 27,1850067

Meisel, Z., Deibel, A., Keek, L., Shternin, P., \& Elfritz, J. 2018, J. Phys. G, 45, 093001

Merritt, R. L., Cackett, E. M., Brown, E. F., et al. 2016, ApJ, 833, 186

Mikles, V., \& Hynes, R. I. 2012, ApJ, 750, 132

Miralda-Escudé, J., Haensel, P., \& Paczyński, B. 1990, ApJ, 362, 572

Mitra, A. 1998, ArXiv e-prints [arXiv:astro-ph/9811402]

Morsink, S. M., \& Leahy, D. A. 2011, ApJ, 726, 56

Nowak, M. A., Heinz, S., \& Begelman, M. C. 2002, ApJ, 573, 778

Oertel, M., Hempel, M., Klahn, T., \& Typel, S. 2017, Rev. Mod. Phys., 89, 015007
Ootes, L. S., Page, D., Wijnands, R., \& Degenaar, N. 2016, MNRAS, 461, 4400 Ootes, L. S., Wijnands, R., Page, D., \& Degenaar, N. 2018, MNRAS, 477, 2900 Ootes, L. S., Vats, S., Page, D., et al. 2019, MNRAS, 487, 1447 Ortolani, S., Barbuy, B., Bica, E., et al. 1999, A\&A, 350, 840

Ortolani, S., Barbuy, B., Bica, E., Zoccali, M., \& Renzini, A. 2007, A\&A, 470, 1043

Page, D., Lattimer, J. M., Prakash, M., \& Steiner, A. W. 2013, ArXiv e-prints [arXiv:1302.6626]

Parikh, A. S., \& Wijnands, R. 2017, MNRAS, 472, 2742

Papitto, A., D'Aì, A., Motta, S., et al. 2011, A\&A, 526, L3

Papitto, A., Ferrigno, C., Bozzo, E., et al. 2013, Nature, 501, 517

Parikh, A. S., Homan, J., Wijnands, R., et al. 2017, ApJ, 851, L28

Parikh, A. S., Wijnands, R., Degenaar, N., Ootes, L., \& Page, D. 2018, MNRAS, 476, 2230

Parmar, A. N., White, N. E., Giommi, P., \& Gottwald, M. 1986, ApJ, 308, 199

Pearson, J. M., Chamel, N., Potekhin, A. Y., et al. 2018, MNRAS, 482, 2994, Erratum: 2019, MNRAS, 486, 768

Ponti, G., Bianchi, S., Muños-Darias, T., \& Nandra, K. 2018, MNRAS, 481, L94

Potekhin, A. Y., \& Chabrier, G. 2013, A\&A, 550, A43

Potekhin, A. Y., \& Chabrier, G. 2018, A\&A, 609, A74

Potekhin, A. Y., Chabrier, G., \& Yakovlev, D. G. 1997, A\&A, 323, 415

Potekhin, A. Y., Pons, J. A., \& Page, D. 2015, Space Sci. Rev., 191, 239

Richardson, M. B., Van Horn, H. M., \& Savedoff, M. P. 1979, ApJS, 39, 29

Riggio, A., Di Salvo, T., Burderi, L., et al. 2008, ApJ, 678, 1273

Rivera Sandoval, L. E., Wijnands, R., Degenaar, N., et al. 2018, MNRAS, 479, 2777

Rutledge, R. E., Bildsten, L., Brown, E. F., Pavlov, G. G., \& Zavlin, V. E. 1999, ApJ, 514, 945

Sanna, A., Burderi, L., Riggio, A., et al. 2016, MNRAS, 459, 1340

Sato, K. 1979, Prog. Theor. Phys., 62, 957

Schaab, C., Voskresensky, D., Sedrakian, A. D., Weber, F., \& Weigel, M. K. 1997, A\&A, 321, 591

Schmitt, A., \& Shternin, P. 2018, in The Physics and Astrophysics of Neutron Stars, eds. L. Rezzolla, P. Pizzochero, D. I. Jones, N. Rea, \& I. Vidaña (Switzerland: Springer), Astrophys. Space Sci. Lib., 457, 455

Shternin, P. S., Baldo, M., \& Haensel, P. 2018, Phys. Lett. B, 786, 28

Sedrakian, A., \& Clark, J.W. 2018, ArXiv e-prints [arXiv:1802.00017]

Tailo, M., D’Antona, F., Burderi, L., et al. 2018, MNRAS, 479, 817

Thorne, K. S. 1977, ApJ, 212, 825

Tomsick, J. A., Gelino, D. M., \& Kaaret, P. 2005, ApJ, 635, 1233

Tomsick, J. A., Gelino, D. M., \& Kaaret, P. 2007, ApJ, 663, 461

Torres, M. A. P., Jonker, P. G., Steeghs, D., et al. 2008, ApJ, 672, 1079

Turlione, A., Aguilera, D. N., \& Pons, J. A. 2015, A\&A, 577, A5

Van, K. X., Ivanova, N., \& Heinke, C. O. 2019, MNRAS, 483, 5595

Vats, S., Wijnands, R., Parikh, A. S., et al. 2018, MNRAS, 477, 2494

Voskresensky, D. N. 2001, in Physics of Neutron Star Interiors, eds. D. Blaschke, N. K. Glendenning, \& A. Sedrakian, Lect. Notes Phys., 578, 467

Walsh, A. R., Cackett, E. M., \& Bernardini, F. 2015, MNRAS, 449, 1238

Wang, Z., Breton, R., Heinke, C. O., Deloye, C., \& Zhong, J. 2013, ApJ, 765, 151

Wang, L., Steeghs, D., Casares, J., et al. 2017, MNRAS, 466, 2261

Watts, A. L. 2012, ARA\&A, 50, 609

Watts, A. L., Krishnan, B., Bildsten, L., \& Schutz, B. F. 2008, MNRAS, 389, 839

Wei, J.-B., Burgio, G. F., \& Schulze, H.-J. 2019, MNRAS, 484, 5162

Wijnands, R., Degenaar, N., \& Page, D. 2013, MNRAS, 432, 2366

Wijnands, R., Degenaar, N., \& Page, D. 2017, J. Astrophys. Astron., 38, 49

Wiringa, R. B., Stoks, V. G. B., \& Schiavilla, R. 1995, Phys. Rev. C, 51, 38

Yakovlev, D. G., Kaminker, A. D., Gnedin, O. Y., \& Haensel, P. 2001, Phys. Rep., 354, 1

Yakovlev, D. G., Levenfish, K. P., \& Haensel, P. 2003, A\&A, 407, 265

Yakovlev, D. G., Levenfish, K. P., Potekhin, A. Y., Gnedin, O. Y., \& Chabrier, G. 2004, A\&A, 417, 169 